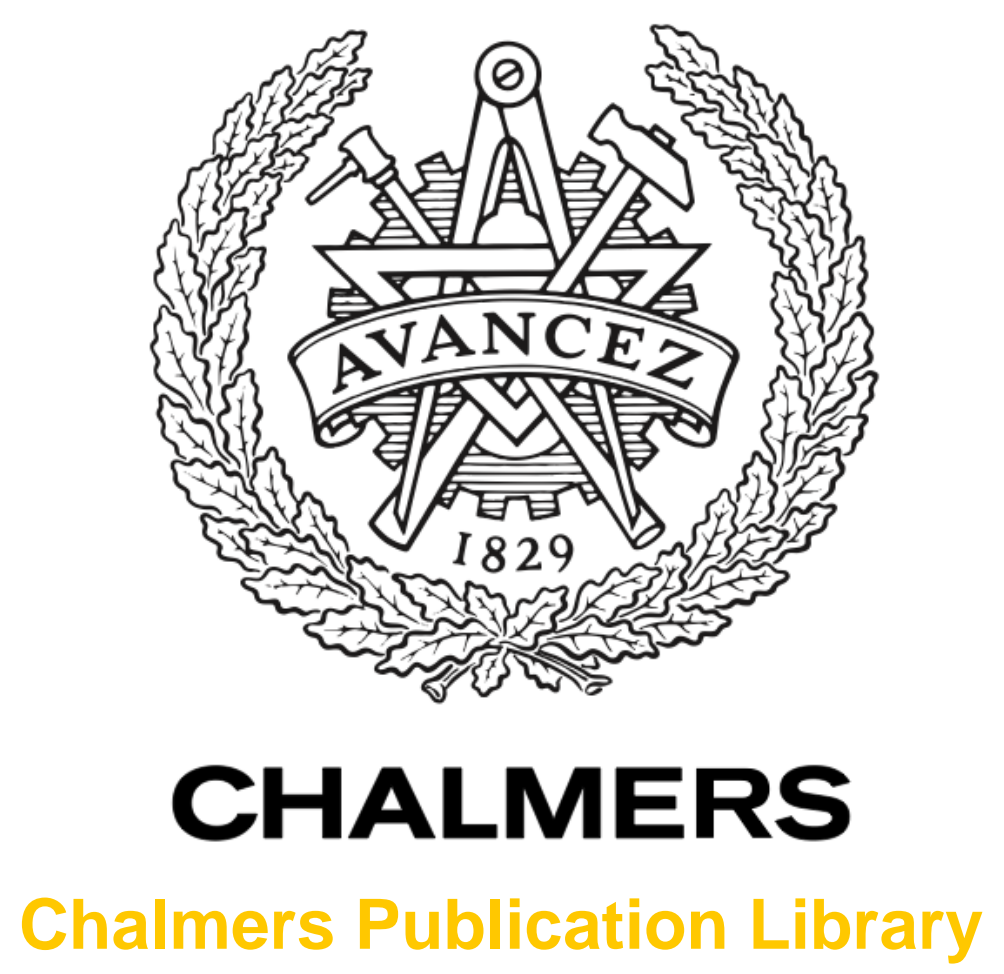

Integrated evasive manoeuvre assist for collision mitigation with oncoming vehicles

This document has been downloaded from Chalmers Publication Library (CPL). It is the author's version of a work that was accepted for publication in:

Vehicle System Dynamics (ISSN: 0042-3114)

Citation for the published paper:

Arikere, A. ; Yang, D. ; Klomp, M. et al. (2018) "Integrated evasive manoeuvre assist for collision mitigation with oncoming vehicles". Vehicle System Dynamics pp. 1-27.

http://dx.doi.org/10.1080/00423114.2017.1423091

Downloaded from: http://publications.lib.chalmers.se/publication/254461

Notice: Changes introduced as a result of publishing processes such as copy-editing and formatting may not be reflected in this document. For a definitive version of this work, please refer to the published source. Please note that access to the published version might require a subscription.

Chalmers Publication Library (CPL) offers the possibility of retrieving research publications produced at Chalmers University of Technology. It covers all types of publications: articles, dissertations, licentiate theses, masters theses, conference papers, reports etc. Since 2006 it is the official tool for Chalmers official publication statistics. To ensure that Chalmers research results are disseminated as widely as possible, an Open Access Policy has been adopted.

The CPL service is administrated and maintained by Chalmers Library. 
To appear in Vehicle System Dynamics Vol. 00, No. 00, Month 20XX, 1-28

\title{
Integrated Evasive Manoeuvre Assist for Collision Mitigation with Oncoming Vehicles
}

\author{
Adithya Arikere ${ }^{\text {ad* }}$, Derong Yang ${ }^{\mathrm{b}}$, Matthijs Klomp ${ }^{\text {cd }}$ and Mathias Lidberg ${ }^{\mathrm{d}}$ \\ ${ }^{a}$ American Axle \& Manufacturing, Inc, \\ ${ }^{b}$ Volvo Car Corporation, \\ Trollhättan Technical Center, \\ Gunnar W Anderssons Passage 25, \\ SE-46153 Trollhättan, Sweden \\ adithya.arikere@aam.com \\ $+46725501589$ \\ Vehicle Motion \& Control, \\ Gunnar Engellaus Väg PVV1:1, \\ SE-405 31 Gothenburg, Sweden \\ derong.yang@volvocars.com \\ $+46723717976$ \\ ${ }^{c}$ Volvo Car Corporation, \\ Vehicle Dynamics CAE, \\ Gunnar Engellaus Väg PVV1:1, \\ SE-405 31 Gothenburg, Sweden, \\ matthijs.klomp@volvocars.com \\ $+46723717356$

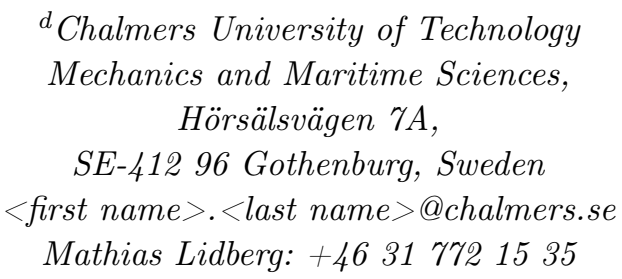

(22nd Dec 2017)

\section{Acknowledgements}

The authors would like to gratefully acknowledge the contributions of Bengt Jacobson (Chalmers), Gunnar Olsson ( $\AA F$ ), Rickard Nilsson (Volvo Cars) and Torbjörn Norlander (NEVS) towards this project and paper. We would also like to thank Volvo Cars for providing the test vehicle, measurement equipment, test track time and the simulation model for use in this project. Additionally, the support of Vector, IPG and Tomlab in providing educational licenses for the measurement, simulation and optimal control software respectively is gratefully acknowledged. Finally, we would like to acknowledge the support of our project partner American Axle and Manufacturing (AAM).

\section{Funding}

We would like to thank Vinnova of the Fordonsstrategisk Forskning och Innovation (FFI), Traffic Safety and Automated Vehicles programme for providing the funding for this project (grant no. 2015-04812).

*Corresponding author 


\begin{abstract}
Development and deployment of steering based collision avoidance systems are made difficult due to the complexity of dealing with oncoming vehicles during the evasive manoeuvre. A method to mitigate the collision risk with oncoming vehicles during such manoeuvres is presented in this work. A point mass analysis of such a scenario is first done to determine the importance of speed for mitigating the collision risk with the oncoming vehicle. A characteristic parameter was identified, which correlates well with the need to increase or decrease speed, in order to reduce the collision risk. This finding was then verified in experiments using a Volvo XC90 test vehicle. A closed-loop longitudinal acceleration controller for collision mitigation with oncoming vehicles is then presented. The longitudinal control is combined with yaw stability control using control allocation to form an integrated controller. Simulations in CarMaker using a validated XC90 vehicle model and the proposed controller showed consistent reductions in the collision risk with the oncoming vehicle.
\end{abstract}

Keywords: Collision avoidance, Integrated motion control, Longitudinal acceleration control, Driver assistance systems, Optimal control

\title{
1. Introduction
}

One of the most common traffic accident types in the world is the rear-end collision [1] which accounts for close to a third of all accidents. While many of these can be prevented with Forward Collision Warning (FCW) or Automatic Emergency Braking (AEB) [2-4], many others require evasive steering [5].

However, development of such steering based autonomous or assist systems are made difficult by the fact that when changing the vehicle trajectory, threats need to be assessed along the potential evasive trajectories as well. Such evasive steering assist systems have been studied in the past [6-9] but are limited in their functionality due to the limited threat assessment accuracy and the underlying system safety challenges. Till date, only a few such assistance systems have been announced by any major OEMs to go on the market $[10,11]$.

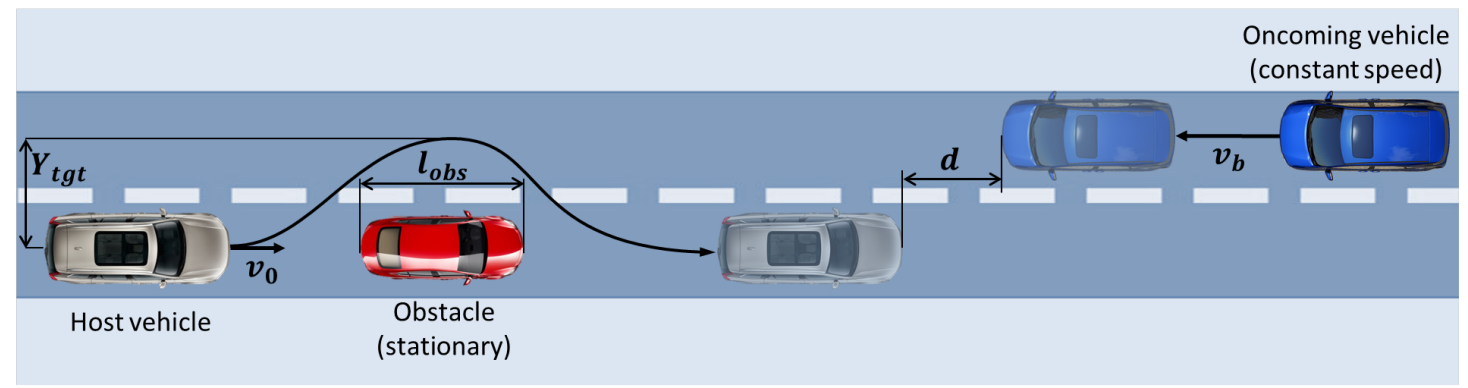

Figure 1 Evasive manoeuvre for collision avoidance in the presence of oncoming vehicles. The distance margin $d$, is a measure of the risk of collision with the oncoming vehicle.

It is clear that unknown threats in the vehicle's evasive trajectory are still a hindrance to the development of such evasive steering functions. One common and primary threat is the oncoming vehicle. Reliable detection of such threats in advance can be challenging since there might not be a clear line of sight to said threat. While technologies like Vehicle-to-Vehicle (V2V) and Vehicle-to-Infrastructure (V2I) communication may help early detection of such threats in the future, it is assumed here that they are detected once the host vehicle clears the obstacle and enters the adjacent lane. In this work, we assume that the driver takes the decision to continue the evasive manoeuvre by passing the obstacle. The controller here aims to solve the complex vehicle dynamics control problem in order to mitigate the collision risk with the oncoming vehicle, directly after it is seen by the sensors on board. 
The issue of dealing with oncoming vehicles has been investigated in the past but in the context of overtaking a moving vehicle [12-16]. However, they mainly focus on decision making, i.e., whether to warn the driver or terminate the manoeuvre, etc. whereas in our use case, we are primarily interested in performing on-line vehicle dynamic control given that the driver has already initiated the manoeuvre. Furthermore, they deal with dynamics that are in the linear range of the tyre characteristics, whereas in our use case, almost all manoeuvres involve significant non-linear tyre and vehicle dynamics. Lastly, they deal with oncoming vehicles in the context of overtaking whereas in our use case, the context is evasive manoeuvres.

In summary, the task of controlling the vehicle motion during emergency steering manoeuvres, particularly with regard to vehicle speed, to mitigate collisions with oncoming vehicles has not been dealt with in the past. The present work in this paper aims to address this issue.

In this work, we find through analytical study, open-loop optimizations and experimental tests, that appropriate control of speed through this manoeuvre can reduce the risk of collision with the oncoming vehicle. However, traditional active safety systems (electronic stability control, roll mitigation, etc.) always reduce speed as a side effect which, in some cases of this accident scenario, can be less than optimal for reducing collision risk with an oncoming vehicle. Our proposed solution to this is an integrated controller that performs both longitudinal acceleration and yaw stability control to better balance the oncoming vehicle collision risk against loss of control.

The accident scenario considered here is illustrated in Figure 1. The distance margin $d$ here, is defined as the distance between the host and the oncoming vehicle at the end of the manoeuvre. This metric is used in the following as a measure of the collision risk with the oncoming vehicle and is used to evaluate the performance of the controller. The end of the manoeuvre is defined as the time instant when the host vehicle has fully returned to the original lane after passing the obstacle.

\section{Manoeuvre Analysis using a Point Mass Model}

To understand how the vehicle needs to be controlled in this scenario, the dynamics of the manoeuvre first needs to be understood. For this purpose, the manoeuvre is first analysed using a point mass model. The point mass model is chosen so as to keep things simple which enables the analysis to be performed analytically. Next, the same model is used in an optimal control framework to verify the results obtained in the analytical analysis. The choice of point mass model here allows a large number of simulations to be run quickly that span a wide range of the parameter space. Lastly, experiments are conducted using a Volvo XC90 test vehicle to validate the results from the point mass analyses. No controller is implemented for these tests. Instead, the driver controls the vehicle manually through the manoeuvre using different strategies informed by the point mass analysis results.

\subsection{The Influence of Speed on Distance Margin to the Oncoming Vehicle}

For a preliminary understanding as to which parameters are likely to be most important for reducing distance margin, a simple analytical analysis using a point mass model was done. A simplified path was assumed for the point mass model as shown in Figure 2. Here, the vehicle is assumed to travel with a constant global $X$ velocity $v_{0}$ and is assumed to be able to reach peak global $Y$ acceleration instantaneously. Small course angles are assumed and the sections 1,2 and 4,5 are assumed to be symmetric. 


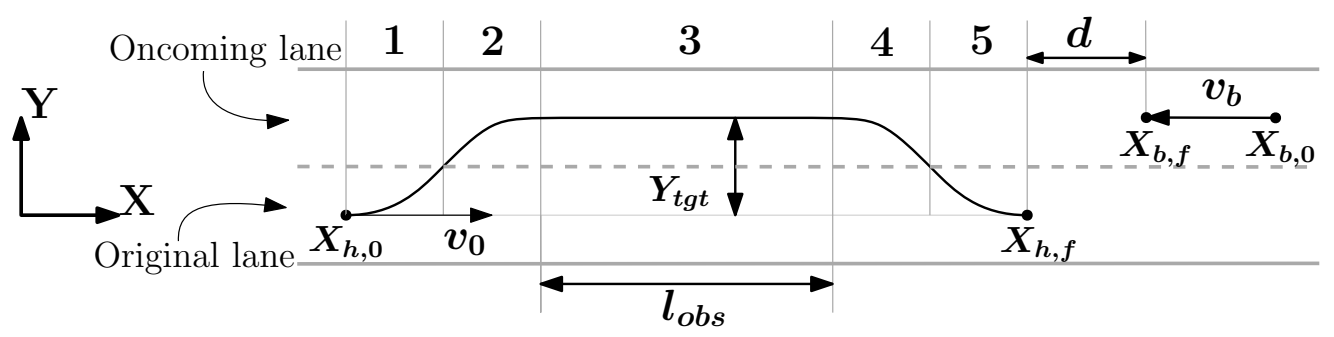

Figure 2 A simplified path that the point mass takes through the manoeuvre

Since the sections 1 and 2 are symmetric, the lateral displacement at the end of section 1 will be $Y_{t g t} / 2$. Assuming a global $Y$ acceleration of $\mu g$, the time to complete section 1 can be given by:

$$
t_{1}=\sqrt{\frac{Y_{t g t}}{\mu g}}
$$

Since sections 1, 2, 4 and 5 are symmetric and the time for the straight section is simply $l_{o b s} / v_{0}$, the time required for the vehicle to complete the entire manoeuvre is given by:

$$
T=4 \sqrt{\frac{Y_{t g t}}{\mu g}}+\frac{l_{o b s}}{v_{0}}
$$

The distance travelled by the host and oncoming vehicles (also called the bullet vehicle since it is the major threat of concern in this work) over the course of the manoeuvre is therefore (assuming constant longitudinal speeds):

$$
d_{t r}=\left(4 \sqrt{\frac{Y_{t g t}}{\mu g}}+\frac{l_{o b s}}{v_{0}}\right)\left(v_{0}+v_{b}\right)
$$

This metric is directly related to the distance margin $(d)$ as $d=d_{0}-d_{t r}$ where, $d_{0}$ is the initial distance between the host and the bullet vehicles which is constant for a given scenario.

Since the lateral dynamics in this manoeuvre is controlled by the driver and is otherwise constrained by the need to avoid the obstacle and return to the original lane, opportunities to further tune the same for an improvement in the distance margin are likely to be limited. The longitudinal dynamics on the other hand is free from any requirements and could potentially be a route through which distance margin improvements could be achieved. Hence, the longitudinal speed was chosen as a variable of interest for an intervention.

Taking the derivative of expression Equation (3) with respect to speed shows how the importance of speed through this manoeuvre varies with different parameters.

$$
\frac{\mathrm{d} d}{\mathrm{~d} v_{0}}=4 \sqrt{\frac{Y_{t g t}}{\mu g}}-\frac{l_{o b s} v_{b}}{v_{0}^{2}}
$$




\subsection{Optimal Control Analysis Supports the Analytical Result}

In order to verify the analytical result from Equation (4), a large number of simulations are run using a point mass model in an optimal control framework ${ }^{1}$. The manoevre parameters are varied across a wide range to investigate different variations of the manoeuvre (see Table 1) and the constraints on the longitudinal force are varied to investigate the effects of longitudinal force in this scenario. The distance margin $d$, is taken as the objective function to be maximised and the global forces as the control inputs to be optimised. Constraints are added to limit the forces to within the friction circle and for the path to avoid the obstacle and return to the original lane. The optimal control problem is presented as a maximisation of the objective function as follows:

Objective function:

$$
\begin{gathered}
d=d_{0}-\int_{0}^{T}\left(\dot{X}+v_{b}\right) \mathrm{d} t=d_{0}-\left(d_{t r, h}+v_{b} T\right) \\
\text { subj. to: } \\
m \ddot{X}=F_{X}, m \ddot{Y}=F_{Y}, F_{X}^{2}+F_{Y}^{2} \leq(\mu m g)^{2} \\
X(0)=0, Y(0)=0, \dot{X}(0)=v_{0}, \dot{Y}(0)=0 \\
Y(T)=0, \dot{Y}(T)=0
\end{gathered}
$$

The path constraints for the point mass in order to avoid the obstacle are defined as follows:

$$
\begin{aligned}
Y\left(t_{1}\right) \geq\left(Y_{t g t}-0.5\right) & X\left(t_{2}\right)-X\left(t_{1}\right) \geq l_{\text {obs }} \\
Y\left(t_{2}\right) \geq\left(Y_{\text {tgt }}-0.5\right) & 0<t_{1} \leq t_{2}<T
\end{aligned}
$$

The time instants when the point mass passes the rear and front edges of the obstacle are defined as $t_{1}$ and $t_{2}$ respectively. The width of the obstacle is defined to be $\left(Y_{t g t}-0.5\right)$ and the point mass is allowed a lateral position window of $\pm 0.5 \mathrm{~m}$ around $Y_{t g t}$ as it passes the obstacle. This margin is chosen roughly based on a $3 \mathrm{~m}$ wide lane and a $2 \mathrm{~m}$ wide vehicle leaving a total margin of $1 \mathrm{~m}$.

The constraints on the path tangential force to investigate the effect of propulsion and braking are implemented as follows:

$$
\begin{aligned}
-\mu m g & \leq F_{x} \leq \mu m g & & \text { propulsion and braking } \\
-\mu m g & \leq F_{x} \leq 0 & & \text { braking only } \\
0 & \leq F_{x} \leq \mu m g & & \text { propulsion only } \\
0 & \leq F_{x} \leq 0 & & \text { constant speed }
\end{aligned}
$$

where $F_{x}$ is the longitudinal force in the vehicle reference frame and can be expressed as:

$$
\begin{aligned}
& {\left[\begin{array}{l}
F_{x} \\
F_{y}
\end{array}\right]=\left[\begin{array}{cc}
\cos \nu & \sin \nu \\
-\sin \nu & \cos \nu
\end{array}\right]\left[\begin{array}{l}
F_{X} \\
F_{Y}
\end{array}\right]} \\
& \tan \nu=\frac{\dot{Y}}{\dot{X}}
\end{aligned}
$$

\footnotetext{
${ }^{1}$ A MATLAB based optimal control software called PROPT from TOMLAB used for this purpose
} 
The parameters and the variations considered for this investigation are tabulated in Table 1.

Table 1. Parameter variations for the point mass analysis

\begin{tabular}{ll}
\hline Parameter & Variation \\
\hline Host initial velocity $\left(v_{0}\right)$ & {$[40,60, \ldots 120] \mathrm{km} / \mathrm{h}$} \\
Bullet vehicle velocity $\left(v_{b}\right)$ & {$[20,40, \ldots 140] \mathrm{km} / \mathrm{h}$} \\
Obstacle length $\left(l_{\text {obs }}\right)$ & {$[0,5, \ldots 25] \mathrm{m}$} \\
Road surface friction $(\mu)$ & {$[0.3,0.5,0.7,1]$} \\
Lateral displacement $\left(Y_{t g t}\right)$ & {$[1,1.5, \ldots 3.5] \mathrm{m}$} \\
\hline
\end{tabular}

The results from the optimal control simulations were then analysed and a regression analysis done on the distance margin improvement achievable with propulsion (acceleration) over braking (deceleration) versus various sets of parameters as identified from Equation (4).

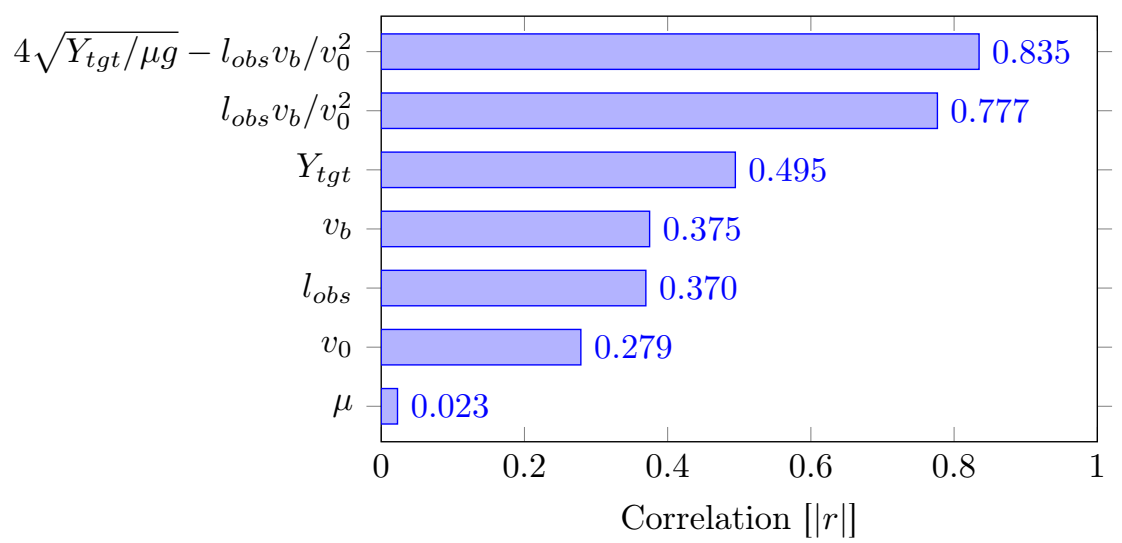

Figure 3 Correlation of manoeuvre parameters to the distance margin benefit that can be achieved with propulsion

As can be seen from the regression analysis results in Figure 3, the parameter with the highest correlation to increased benefit due to propulsion is $\left(4 \sqrt{Y_{t g t} / \mu g}-l_{o b s} v_{b} / v_{0}^{2}\right)$ (henceforth called the characteristic parameter). Some of the reasons why the correlation factor is not higher include the fact that the analytical expression is based on a very simple model that assumes constant global X velocity. Another important effect that is not captured by the analytical model is that the optimal path shows apex hitting behaviour which becomes important when the first part of the expression becomes prominent (large lateral displacement or low friction).

As can be seen, with the simplified path (analytical model), the path can be distinctly split into three major parts where it is avoiding the obstacle (1), passing the obstacle (2) and returning to the original lane (3). With the optimal path on the other hand, the point mass shows apex hitting behaviour and the path is a single cohesive profile and cannot be separated into distinct parts.

In the characteristic parameter, the role of $l_{o b s}$ is immediately apparent. The longer the obstacle, the longer the host vehicle has to travel in the oncoming lane and therefore, the longer is the distance travelled by the two vehicles. Hence speeding up is beneficial in such cases in order to reduce the time spent in the oncoming lane. This effect can be seen in Figure $4 \mathrm{a}$.

However, the role of the oncoming vehicle to host vehicle velocity ratio is less obvious. This is related to the time spent by the host vehicle performing the manoeuvre (see Equation (5)). When the bullet vehicle is travelling relatively fast, the distance it travels 


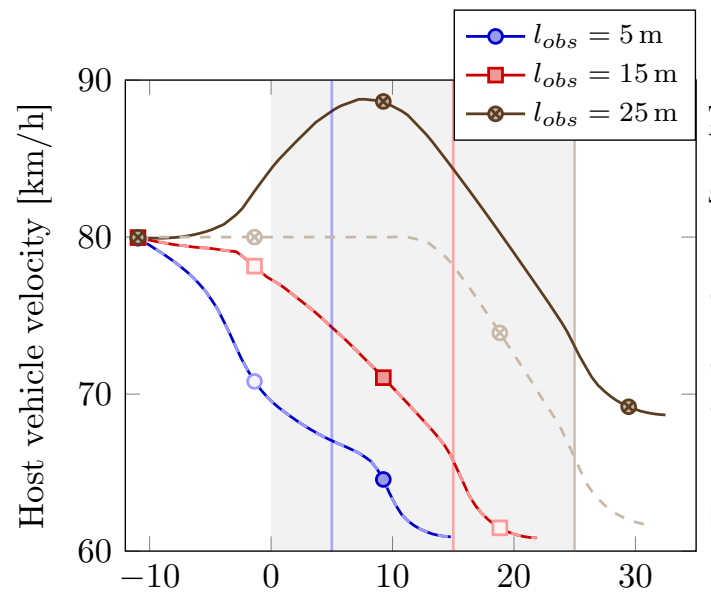

(a) Distance travelled $[\mathrm{m}]$

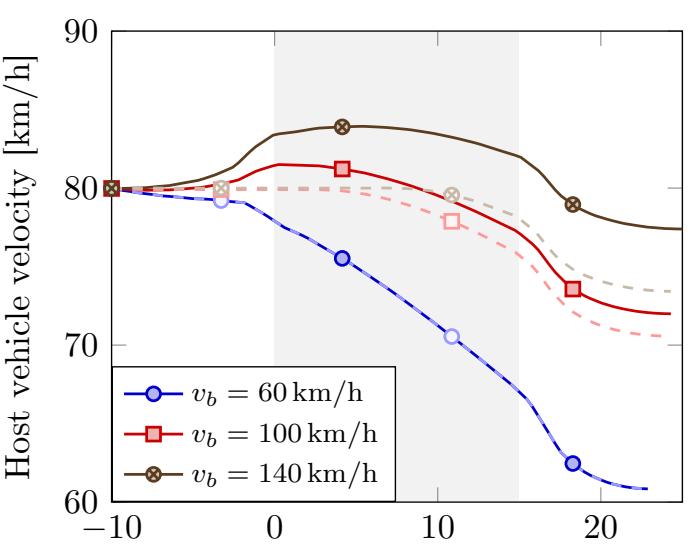

(b) Distance travelled [m]

Figure 4 Host vehicle velocity profiles for a starting speed of $v_{0}=80 \mathrm{~km} / \mathrm{h}$, friction and lateral displacement of $\mu=1$ and $Y_{t g t}=1.5 \mathrm{~m}$ respectively. Bullet vehicle speed of $v_{b}=60 \mathrm{~km} / \mathrm{h}$ (a) and obstacle length of $l_{\text {obs }}=15$ $\mathrm{m}$ (b) are considered. The dashed, greyed out plots indicate cases where positive longitudinal force (propulsion) is not allowed. The greyed out area represents the location of the obstacles. The vertical lines in the greyed out area in (a) represent the ends of the different obstacles.

is relatively large compared to the host vehicle. As a result, it becomes more important for the host vehicle to reduce the duration of the manoeuvre as opposed to reduce the distance it travels. This effect can be seen in the right panel of Figure $4 \mathrm{~b}$ where the host vehicle begins to accelerate (when propulsion is available) when the bullet vehicle is travelling relatively fast.

As can be seen from Figure 4, there are cases in which increasing the speed of the vehicle is beneficial. It can be seen that when the bullet vehicle speed is relatively high, the optimal result favours an increase in speed and vice versa. A similar result is seen with respect to obstacle length as well wherein a longer obstacle results in a speed increase to be favoured. To measure the impact of propulsion in these scenarios, the same simulations were also done without the ability to apply positive longitudinal force (also seen in Figure 4) and the resulting distance margins compared. It was seen that distance margin increases of up to $2 \mathrm{~m}$ could be obtained just by controlling speed in these cases. Note that the cases with propulsion take longer distance to perform the manoeuvre despite which they achieve a larger distance margin.

Assuming a case of emergency avoidance for the obstacle and the oncoming vehicle, the lateral dynamics of the vehicle will already will be near on-limit. As a result, the opportunity for lateral control to improve distance margin in addition to assisting in avoidance and stability is small. However, given that speed plays such a significant role in distance margin and is otherwise unconstrained, integrated control around the theme of controlling vehicle speed could potentially yield large benefits.

\subsection{Experimental Results Confirm the Influence of Speed}

To validate the hypothesis regarding the correlation between speed and the distance margin, experiments were conducted with a Volvo XC90 test vehicle (see Figure 6). The test vehicle is equipped with state-of-the-art yaw and roll stability control systems. It has a gasoline-electric hybrid drivetrain with a primary internal combustion engine (ICE) driving the front axle, and a secondary electric motor driving the rear axle. The scenario specification for the experiments is shown in Table 2. The schematic of the track layout 
for the two cases are shown in Figure 5.

Table 2. Scenario specification for experiments

\begin{tabular}{lccl}
\hline Parameter & Scen. A & Scen. B & Unit \\
\hline Host initial velocity & 55 & 75 & $\mathrm{~km} / \mathrm{h}$ \\
Bullet vehicle velocity & 90 & 30 & $\mathrm{~km} / \mathrm{h}$ \\
Obstacle length & 20 & 0 & $\mathrm{~m}$ \\
Lateral displacement & 3 & 3 & $\mathrm{~m}$ \\
Road surface friction (est) & 0.8 & 0.8 & - \\
\hline
\end{tabular}

The choice of these specific speeds and obstacle lengths is driven partly by the need to generate characteristic parameter values that are well apart to be able to clearly see the influence of speed. It is also partly influenced by practical limitations such as maximum allowed speed on the test track, friction due to poor weather, etc. The initial speed could not be lowered beyond $55 \mathrm{~km} / \mathrm{h}$ since the speed drop due to the stability systems' intervention would result in too low speed at the end.

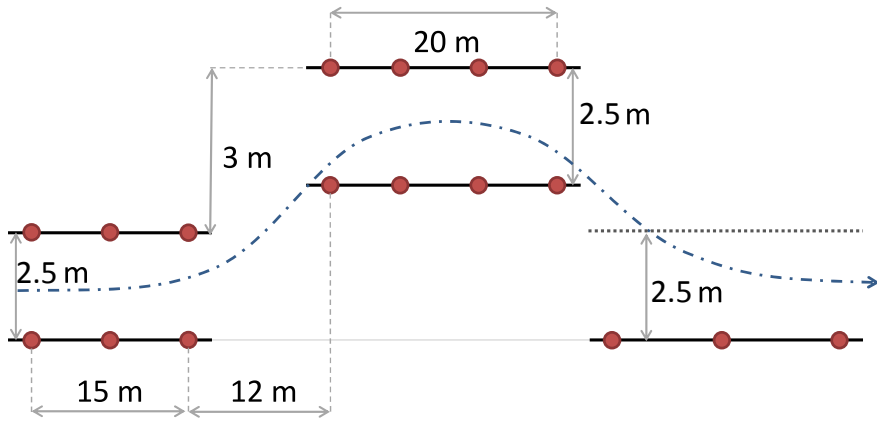

(a) Scenario A

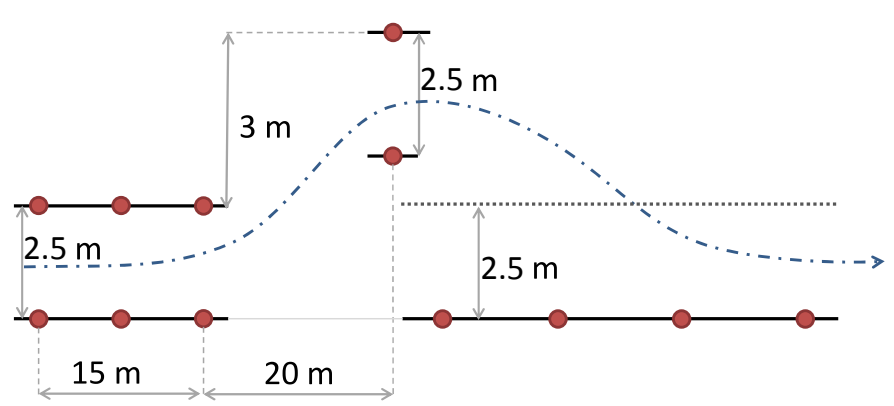

(b) Scenario B

Figure 5 Track layouts for the two experimental scenarios

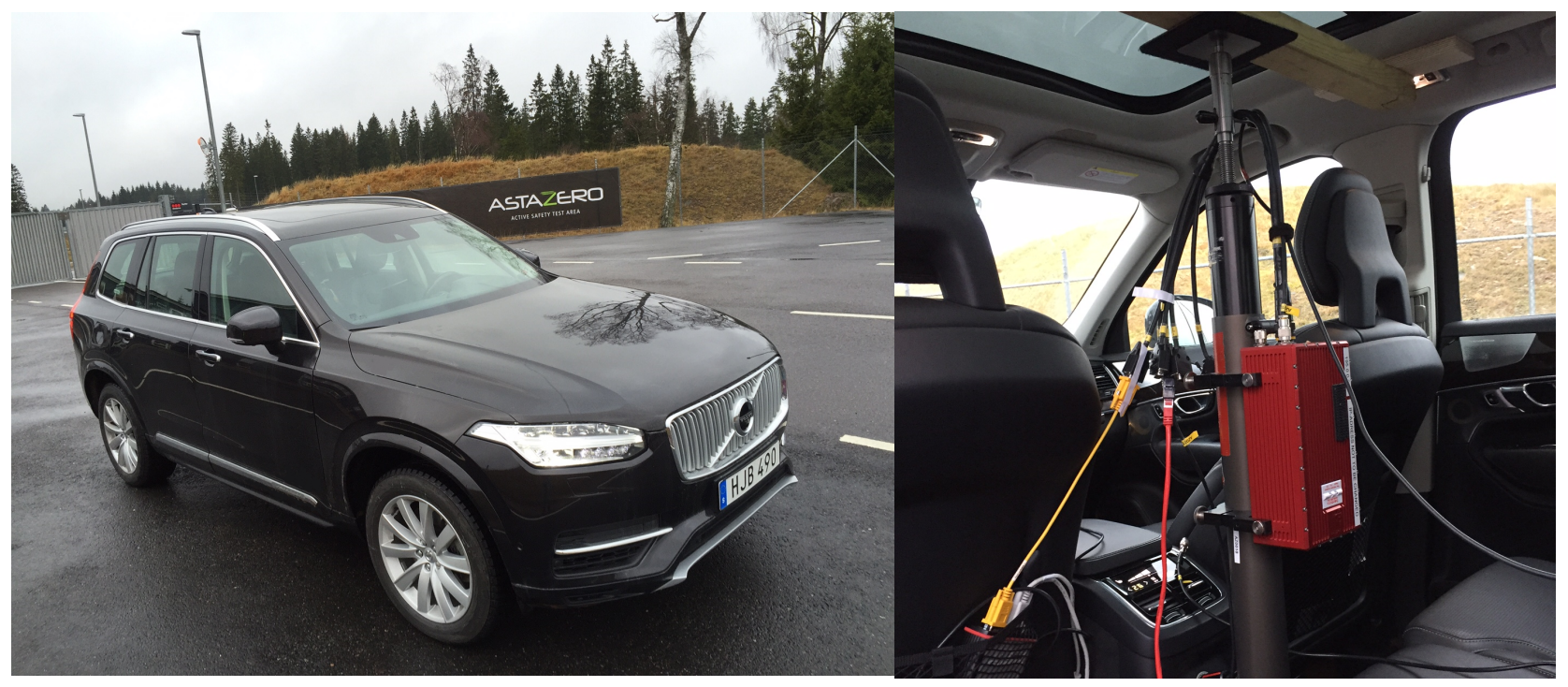

(a) Volvo XC90 test vehicle at the AstaZero proving ground

(b) Instrumented with RT3000 Inertial and GPS unit

Figure 6 The Volvo XC90 T8 test vehicle has a hybrid drivetrain with a 320 hp gasoline engine driving the front axle and a $80 \mathrm{hp}$ electric motor driving the rear axle 
Note that once the host vehicle passes the obstacle, the return lane is not fully defined. The goal of the driver after passing the obstacle is to return to the original lane as quickly as possible. The driver is instructed to get close to the set of cones on the right as quickly as possible after passing the obstacle. The dotted dark grey line represents the left boundary of the return lane. This however is not marked in the actual experiment. The dash-dotted blue lines show a sample vehicle trajectory through these tracks.
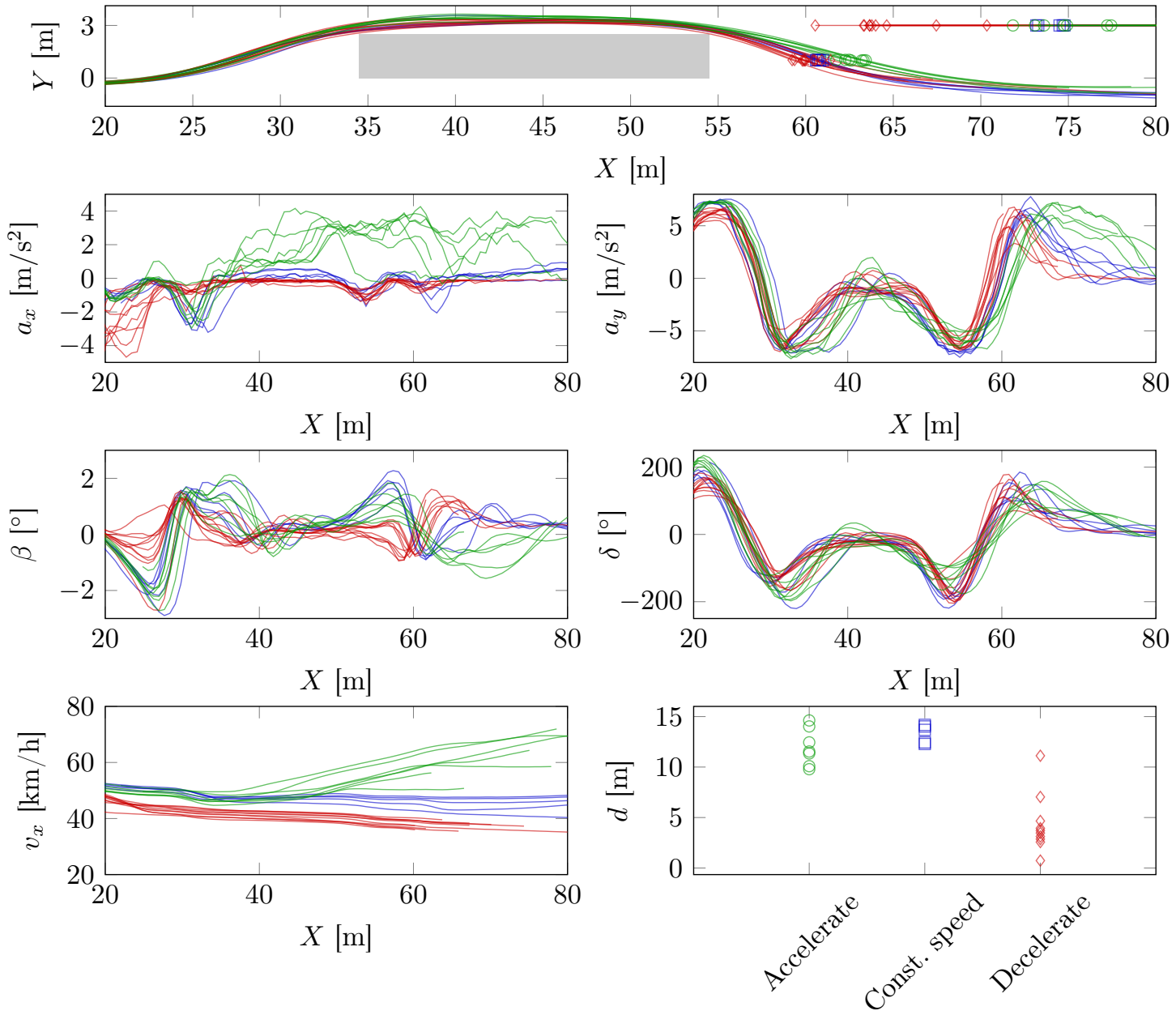

Figure 7 The paths, velocities and the distance margin plots from experiments for Scenario A. The horizontal lines at the top right portion of the path plots show the trajectories and the final positions of the bullet vehicles as the corresponding host vehicles return to the original lane.

The speed through the course of the manoeuvre was manually controlled by the driver. Different strategies to control speed were employed to investigate the effect of speed. In Scenario A, acceleration, constant speed and deceleration were the three strategies that were investigated. In Scenario B, throttle off and deceleration were the two strategies that were investigated. It was not possible to investigate the cases of "constant speed" and "acceleration" due to the manoeuvre already being close to the limit. The lateral control aspect of the vehicle (apart from driver steering) was left to the conventional stability control functions in the vehicle.

Figures 7 and 8 show the results from the test runs, including various vehicle states during the manoeuvres. The path plots also show the location of the obstacle and also the paths of the virtual bullet vehicles travelling at constant speed. Note that the path 

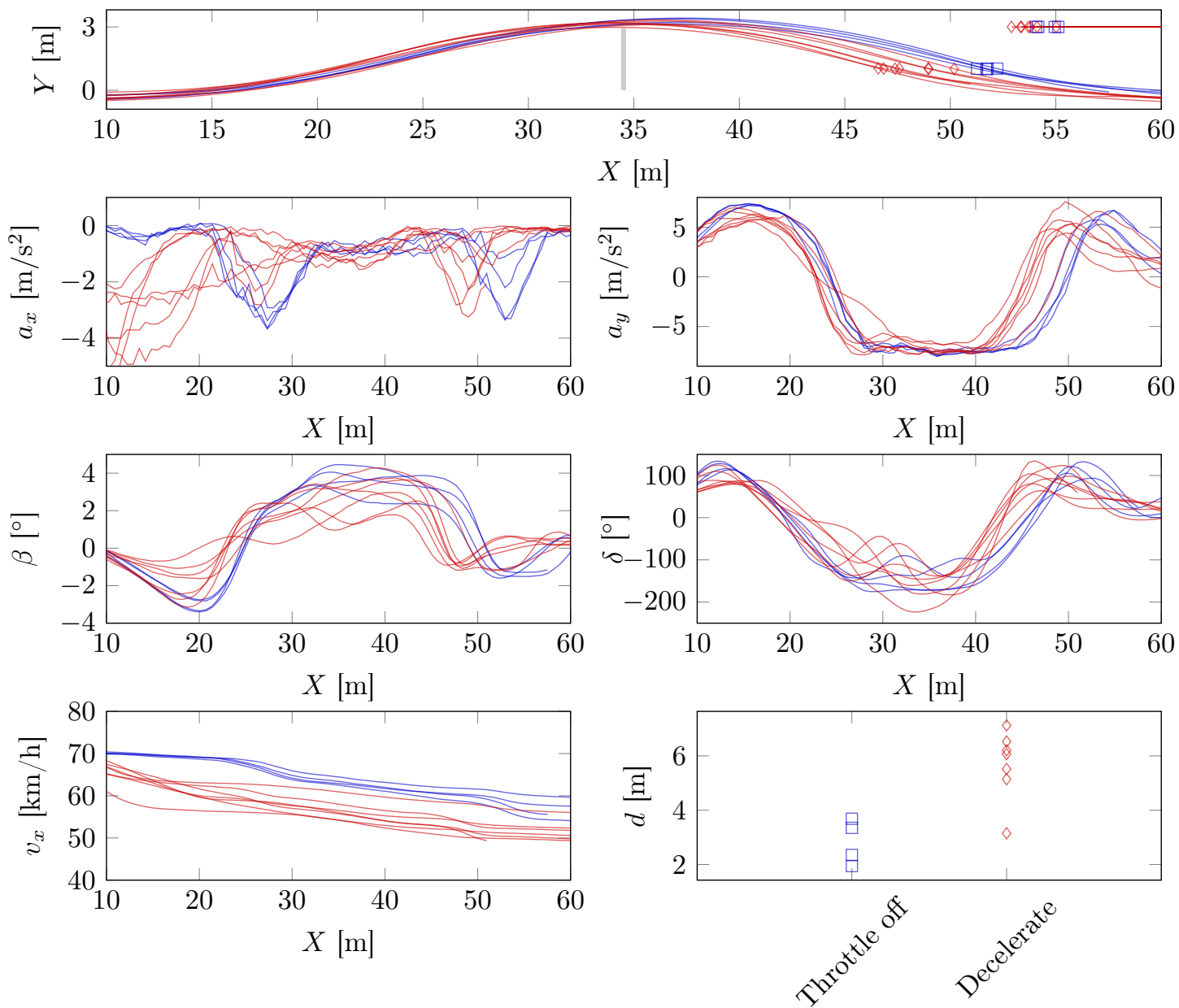

Figure 8 The paths, velocities and the distance margin plots from experiments for Scenario B. The horizontal lines at the top right portion of the path plots show the trajectories and the final positions of the bullet vehicles as the corresponding host vehicles return to the original lane.

and the velocity plots have the same colour coding as the distance margin plots.

From the distance margin plots of Scenario A (Figure 7), it can be seen that accelerating or maintaining speed improves distance margin consistently. Since the characteristic parameter is large in this scenario, this outcome supports the hypothesis. In the case of "constant speed", the speed was controlled with the help of cruise control and hence there is little spread in the resulting distance margins. However, in the case of "Accelerate", the drive torque was cut off by the yaw stability control and hence this resulted in deceleration in the beginning followed by hard acceleration halfway through the manoeuvre. Furthermore, due to the large response times of the engine, the acceleration levels and timings were relatively inconsistent. As a result, a large variation can be seen in the longitudinal acceleration and side slip plots and consequently there is also a large spread in the resulting distance margins for this case. For the "Decelerate" case, two variations of braking were performed: (a) brake hard but only just before the manoeuvre to simulate Collision Mitigation Braking and (b) brake continuously but softly throughout the manoeuvre. Both these interventions were difficult to carry out consistently and hence once again a large spread is seen in the longitudinal acceleration and side slip plots. Consequently a large spread is seen in the distance margin outcomes as well. However, it should be noted that despite the spread in outcomes, the trend is clear that decelerating is worse in this scenario. 
The distance margin plots of Scenario B (Figure 8) show the opposite trends, i.e., braking through the manoeuvre increases the distance margin. Once again, performing the "Decelerate" intervention consistently was difficult and large variation in the longitudinal acceleration and side slip plots can be seen which result in a large spread in the distance margins as well.

Given that the characteristic parameter is small in this case, this outcome also supports the hypothesis. The velocity and the path plots show why lower speed is of benefit in this case. It can be seen in the path plots that when the speed is low (red), the vehicle is able to return to the original lane much more quickly after avoiding the obstacle. This is more important in this scenario than the duration, since the oncoming vehicle is travelling slowly in this case. In Scenario A on the other hand, even though accelerating increases the distance required to complete the manoeuvre, the reduced duration of the same is of larger benefit due to the fast moving oncoming vehicle.

In summary, experimental results support the hypothesis that increasing or maintaining speed in scenarios with large characteristic parameters and vice versa increase the distance margin.

\section{Longitudinal Acceleration Control for Increased Distance Margin}

Using the scheme developed and tested in Section 2.1, a closed loop longitudinal acceleration controller is built here for eventual use with a full vehicle model. In this section, the controller is formulated, implemented and tested with a point mass simulation model.

As part of the online closed loop controller, a scheme to predict the remaining manoeuvre path based on the current vehicle states is presented. This path prediction is based again on a point mass model and simplified dynamics. The goal of this step is to predict a path that the vehicle could potentially take through the manoeuvre and not to follow it. Using this path prediction scheme, an optimal velocity for the vehicle through the manoeuvre is determined in order to maximise the distance margin which in turn is used to determine the longitudinal force to be applied on the vehicle.

This control scheme is then implemented in simulation with a point mass model and tested in the two scenarios defined in Table 2. Point mass optimal control simulations are also performed for the same scenarios and the results compared to that from the closed loop controller.

\subsection{Making a Path Prediction}

Note that while a potential path is predicted in this section, there is no attempt to follow said path. It is simply a path that the vehicle could likely take and is used to estimate the distance margin which is in turn used to perform longitudinal acceleration control.

Since this is an emergency avoidance manoeuvre, the vehicle can be assumed to be operating close to the friction limit while performing the lane changes. The maximum lateral acceleration during the manoeuvre can then be given by $a_{Y}=\mu \mathrm{g}$. Note that if it is known that the vehicle will not be on limit, it is possible to adjust the constructed path by changing the $\mu$ to the expected utilised friction.

The path is constructed assuming that the vehicle achieves the target lateral displacement as soon as possible and returns to original lane as soon as it clears the obstacle (see Figure 9). Constant global X speed and small course angle changes are assumed.

The path is constructed in three sections: first the initial lane change section $P_{0} P_{2}$, next the straight section $P_{2} P_{3}$ and finally the last lane change section $P_{3} P_{5}$. As far as possible, 


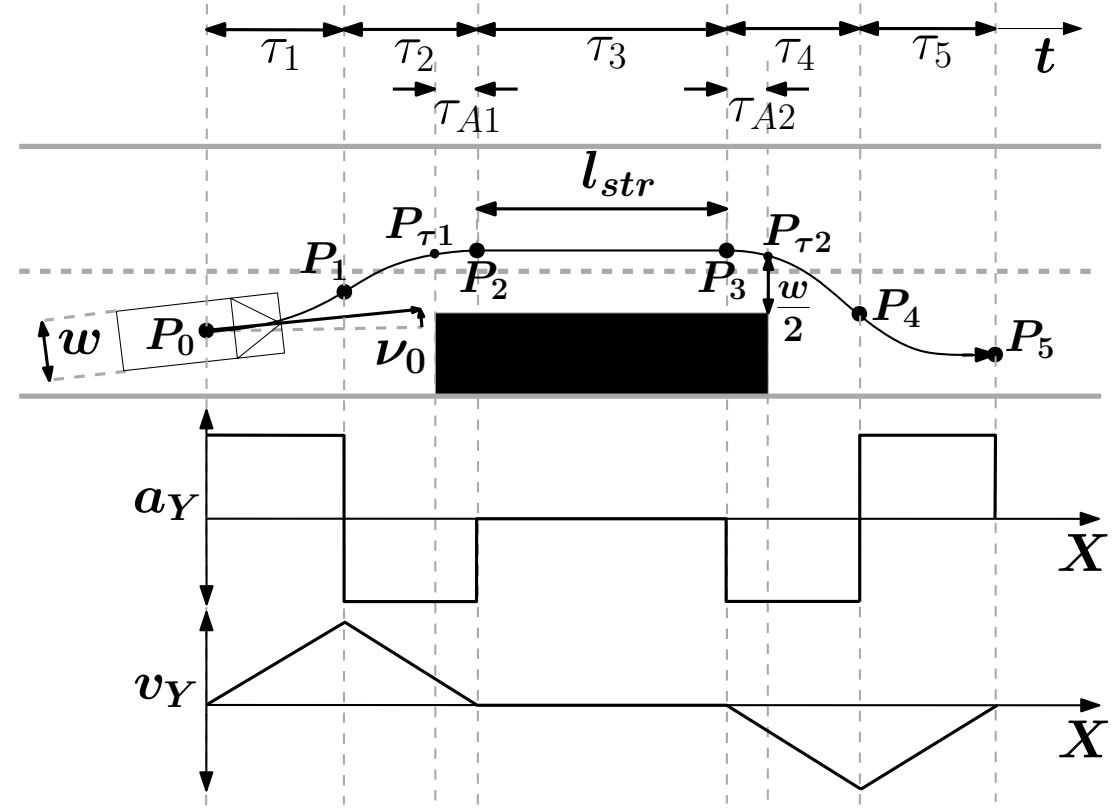

Figure 9 Predicted nominal path construction for longitudinal acceleration control

in the absence of any other requirements, the lane change sections are assumed to be symmetric in their lateral dynamics about their centre points, i.e., the lateral acceleration and lateral velocity profiles of section $P_{0} P_{2}$ and $P_{3} P_{5}$ are symmetric about the points $P_{1}$ and $P_{4}$ respectively. Note that this symmetry is enabled by the constant global $\mathrm{X}$ speed assumption.

As the vehicle travels through the manoeuvre however, and the path needs to be reconstructed, it is no longer possible to assume the path is symmetric. The path needs to be recomputed using different assumptions which are detailed below.

Given a starting position $P_{0}$ and a course angle $\nu_{0}$, the points $P_{1}$ and $P_{2}$ are first determined. The point $P_{1}$ is where the vehicle changes direction and starts turning right $\left(a_{Y}\right.$ changes sign) and $P_{2}$ is when the vehicle has achieved the target lateral displacement and is travelling straight (zero lateral velocity). These conditions are expressed in Equations (13) to (16) and can be used to determine the points $P_{1}$ and $P_{2}$.

$$
\begin{aligned}
v_{Y 1} & =v_{Y 0}+a_{Y} \tau_{1} \\
v_{Y 2} & =v_{Y 1}-a_{Y} \tau_{2}=0 \\
Y_{1} & =Y_{0}+v_{Y 0} \tau_{1}+\frac{1}{2} a_{Y} \tau_{1}^{2} \\
Y_{2} & =Y_{1}+v_{Y 1} \tau_{2}-\frac{1}{2} a_{Y} \tau_{2}^{2}=Y_{t g t}
\end{aligned}
$$

where, $v_{Y 0}=v_{0} \sin \nu_{0}, v_{X}=v_{0}$ (small angle and constant global longitudinal speed assumption) and $v_{0}$ is the current vehicle speed. The points $P_{4}$ and $P_{5}$ are determined using the same method using $P_{2}$ as the starting point.

Next, the time instants, $\tau_{A 1}$ and $\tau_{A 2}$ are determined such that at $\tau_{A 1}$, the vehicle passes the trailing edge of the obstacle and at $\tau_{A 2}$, the path is a distance $w / 2$ (half-track width) 
away from the edge of the obstacle laterally (Equations (17) and (18)).

$$
\begin{aligned}
X_{2} & =X_{o b s, 1}+\tau_{A 1} v_{X} \\
Y_{A 2} & =Y_{2}-\frac{1}{2} a_{Y} \tau_{A 2}^{2}
\end{aligned}
$$

Finally, the length of the straight section is determined such that when added between $P_{2}$ and $P_{3}$, would result in the vehicle passing the leading edge of the obstacle at $P_{\tau 2}$.

The expressions for the parameters so determined in order to construct the path are summarised in Table 3.

\begin{tabular}{|c|c|c|c|}
\hline Parameter & $X_{0}<X_{o b s, 1}$ & $X_{o b s, 1} \leq X_{0}<X_{o b s, 2}$ & $X_{o b s, 2} \leq X_{0}$ \\
\hline \multirow[b]{2}{*}{$\tau_{1}$} & $-2 v_{Y 0}+\sqrt{2 v_{Y 0}^{2}+4 a_{Y}\left(Y_{t g t}-Y_{0}\right)}$ & \multirow[b]{2}{*}{0} & \multirow[b]{2}{*}{0} \\
\hline & $\begin{array}{c}2 a_{Y} \\
\end{array}$ & & \\
\hline$\tau_{2}$ & $\frac{v_{Y 0}}{a_{Y}}+\tau_{1}$ & $\frac{v_{Y}}{a_{Y}}$ & 0 \\
\hline \multirow[b]{2}{*}{$\tau_{A 1}$} & $\underline{X_{2}-X_{o b s, 1}}$ & $X_{2}-X_{0}$ & \multirow{2}{*}{0} \\
\hline & $\overline{v_{X}}$ & $v_{X}$ & \\
\hline$l_{\text {str }}$ & $l_{o b s}+\left(X_{o b s, 1}-X_{A 1}\right)-\left(\tau_{A 1}+\tau_{A 2}\right) v_{X}$ & $l_{o b s}+\left(X_{o b s, 1}-X_{0}\right)-\tau_{A 2} v_{X}$ & 0 \\
\hline \multirow{2}{*}{$\tau_{A 2}$} & $\sqrt{\frac{2\left(Y_{2}-\left(Y_{t g t}-w / 2\right)\right)}{2}}$ & $\sqrt{\frac{2\left(Y_{2}-\left(Y_{t g t}-w / 2\right)\right)}{2}}$ & \multirow{2}{*}{0} \\
\hline & $\sqrt{a_{Y}}$ & $\sqrt{a_{Y}}$ & \\
\hline \multirow{2}{*}{$\tau_{4}$} & $\sqrt{Y_{2}}$ & $-2 v_{Y 0}-\sqrt{2 v_{Y 0}^{2}+4 a_{Y} Y_{2}}$ & $-2 v_{Y 0}-\sqrt{2 v_{Y 0}^{2}+4 a_{Y} Y_{0}}$ \\
\hline & $\sqrt{a_{Y}}$ & $2 a_{Y}$ & $2 a_{Y}$ \\
\hline$\tau_{5}$ & $\sqrt{\frac{Y_{2}}{a_{Y}}}$ & $-\frac{v_{Y 2}}{a_{Y}}+\tau_{4}$ & $-\frac{v_{Y 0}}{a_{Y}}+\tau_{4}$ \\
\hline
\end{tabular}

Table 3. Path parameters

Note that the $X$ position is simply determined as:

$$
\begin{aligned}
X(t) & =X_{0}+v_{X} t \quad \text { where, } t \in[0, T] \\
T & =\Sigma_{i=1}^{5} \tau_{i}
\end{aligned}
$$

The path is continuously reconstructed over the course of the manoeuvre in order to account for the deviations in the actual path that invariably occur due to driver steering.

\subsection{From Predicted Path to Longitudinal Force Demand}

Once the path is predicted, it is then used to estimate the distance margin that will be achieved assuming the host vehicle follows the path at the current speed and the bullet vehicle also travels straight holding its current speed.

$$
J=d=d_{0}-\underbrace{\left(d_{h, X}+\frac{d_{h, X}}{\dot{X}} v_{b}\right)}_{d_{t r}}
$$

where, $d_{0}$ is the current distance between the host vehicle and the bullet vehicle and $d_{h, X}$ is the length of the predicted path along the $X$ axis. The distance travelled by the two vehicles over the course of the manoeuvre is indicated as $d_{t r}$. Both the host and 
the bullet vehicle are assumed to travel at constant speeds in this estimation. Note that the equation becomes undefined when $\dot{X}$ approaches zero and represents the case when the host vehicle has stopped, possibly in the oncoming lane. However, since the distance margin becomes smaller when $\dot{X}$ reduces and since the goal is to maximise the distance margin, the controller should prevent such a situation from ever arising.

The assumption of constant host and bullet vehicle speeds are made partly to keep the controller computationally simple. Non-constant longitudinal speed for the host vehicle would mean the friction circle would have to be taken into account and the changing speed through the manoeuvre would mean it would no longer be possible to take advantage of symmetries in the planned path. The constant bullet vehicle speed assumption is also motivated by the fact that it is difficult to know how the bullet vehicle will react in advance. Instead, due to the simplicity of the current scheme, the objective function can be updated at each time step with the current measured bullet vehicle velocity. If needed however, the objective function can easily be extended to incorporate acceleration profiles for the bullet vehicle by modifying the last term of the $d_{t r}$ expression.

The distance margin, $d$ here is the objective that we intend to maximise by controlling the host vehicle speed. Hence the derivative of $d$ with respect to the host vehicle speed, $\dot{X}$ should give an indication whether we need to increase or decrease vehicle speed to reduce the risk of collision with the oncoming vehicle. Since $d_{0}$ is a constant here, the expression for the derivative can be simplified as follows:

$$
J_{\dot{X}}=\frac{\mathrm{d} d}{\mathrm{~d} \dot{X}}=-\frac{\mathrm{d}}{\mathrm{d} \dot{X}}\left(d_{t r}(\dot{X})\right)
$$

A gradient descent search is then performed until the optimal speed $\dot{X}_{\text {opt }}$ is found. The optimal speed is then used to determine the longitudinal force to be applied assuming that the optimal speed is achieved halfway through the manoeuvre.

$$
F_{x, t g t}=m \frac{\dot{X}_{o p t}-\dot{X}}{T / 2}
$$

This controller was first tested out in a point mass model and compared to the equivalent closed loop simulations. The lateral controller used in these closed loop simulations are taken from [17]. The resulting force demand from the longitudinal and lateral controllers are then allocated to the point mass directly if possible and if not, they are allocated in proportion to their magnitudes but limited by the available road friction.

Figures 10 and 11 show results from the optimal control and the closed loop simulations using the point mass models for scenarios A and B.

From Figure 10, it can be seen that even though the longitudinal acceleration profiles show some dissimilarity, the closed loop profile shows similar trends as the optimal. This can be explained by the fact that the closed loop control is based on a simplified path prediction which is different from the optimal as explained in Section 2.2. Additionally, the lateral control dynamics is not taken into account in the longitudinal control and these two can interfere to create a suboptimal result.

Despite the small dissimilarities seen in the longitudinal acceleration profiles, it can be seen that the velocity, course angle, path and lateral acceleration profiles match closely.

Similarly, in Figure 11, while some dissimilarity is seen in the longitudinal acceleration profiles, the velocity profiles match much more closely. However, in this case, the course angle and the path plots diverge towards the end of the manoeuvre. This is partly due to 

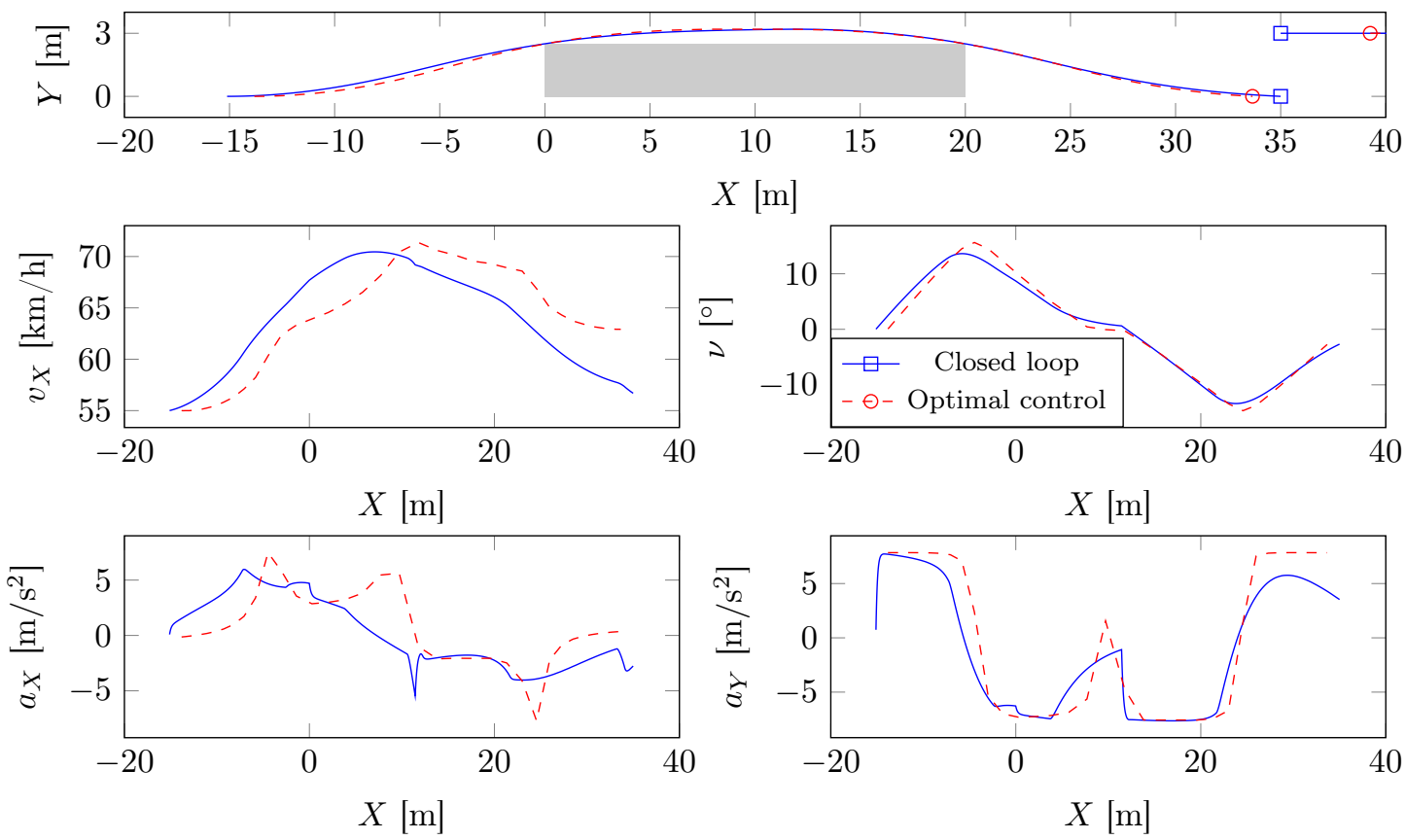

Figure 10 Comparison between closed loop and optimal control for the point mass model in Scenario A. The markers in the path plot represent the location of the vehicles at the end of the manoeuvre.

the fact that as the vehicle speed drops, the maximum allowed course angle rate becomes very sensitive to changes in vehicle speed $\left(\dot{\nu}_{\max }=a_{y, \max } / v_{x}\right)$. Another reason for this divergence is that the lateral control is less aggressive at lower speeds and resulting in a divergence in the lateral acceleration profiles at the end (and consequently the path and course angle as well).

Table 4. Distance margin for the optimal control and closed loop controllers using the point mass models

\begin{tabular}{lcccccc}
\hline & \multicolumn{3}{c}{ Scenario A } & \multicolumn{3}{c}{ Scenario B } \\
& NC & LAC & $\boldsymbol{\Delta}$ & NC & LAC & $\boldsymbol{\Delta}$ \\
\hline Closed loop & -3.4 & 0 & $\mathbf{3 . 4}$ & -13.0 & 0 & $\mathbf{1 3 . 0}$ \\
Optimal control & 1.6 & 5.6 & $\mathbf{4 . 0}$ & -0.7 & 7.8 & $\mathbf{8 . 5}$ \\
$\boldsymbol{\Delta}$ & $\mathbf{5 . 0}$ & $\mathbf{5 . 6}$ & & $\mathbf{1 2 . 3}$ & $\mathbf{7 . 8}$ & \\
\hline NC = No controller, LAC $=$ Longitudinal acceleration controller
\end{tabular}

Table 4 shows the distance margins from the closed loop and the optimal control simulations for the two scenarios. Note that the distance margins have been normalised to the case of closed loop cases (steering) with longitudinal acceleration control. It can be seen that while the closed loop simulations don't perform as well as the optimal, addition of the longitudinal acceleration control consistently increases the distance margin (the $\Delta$ columns). In the case of scenario B, addition of the longitudinal acceleration controller brings the closed loop performance much closer to the optimal (the $\Delta$ row).

\section{Integrated Controller for Robust Distance Margin Improvement}

The longitudinal acceleration controller presented in Section 3 is combined with a yaw stability controller to create an integrated controller (IC) in this section. A simple electronic stability controller (ESC) is used as an example of a yaw stability controller. 

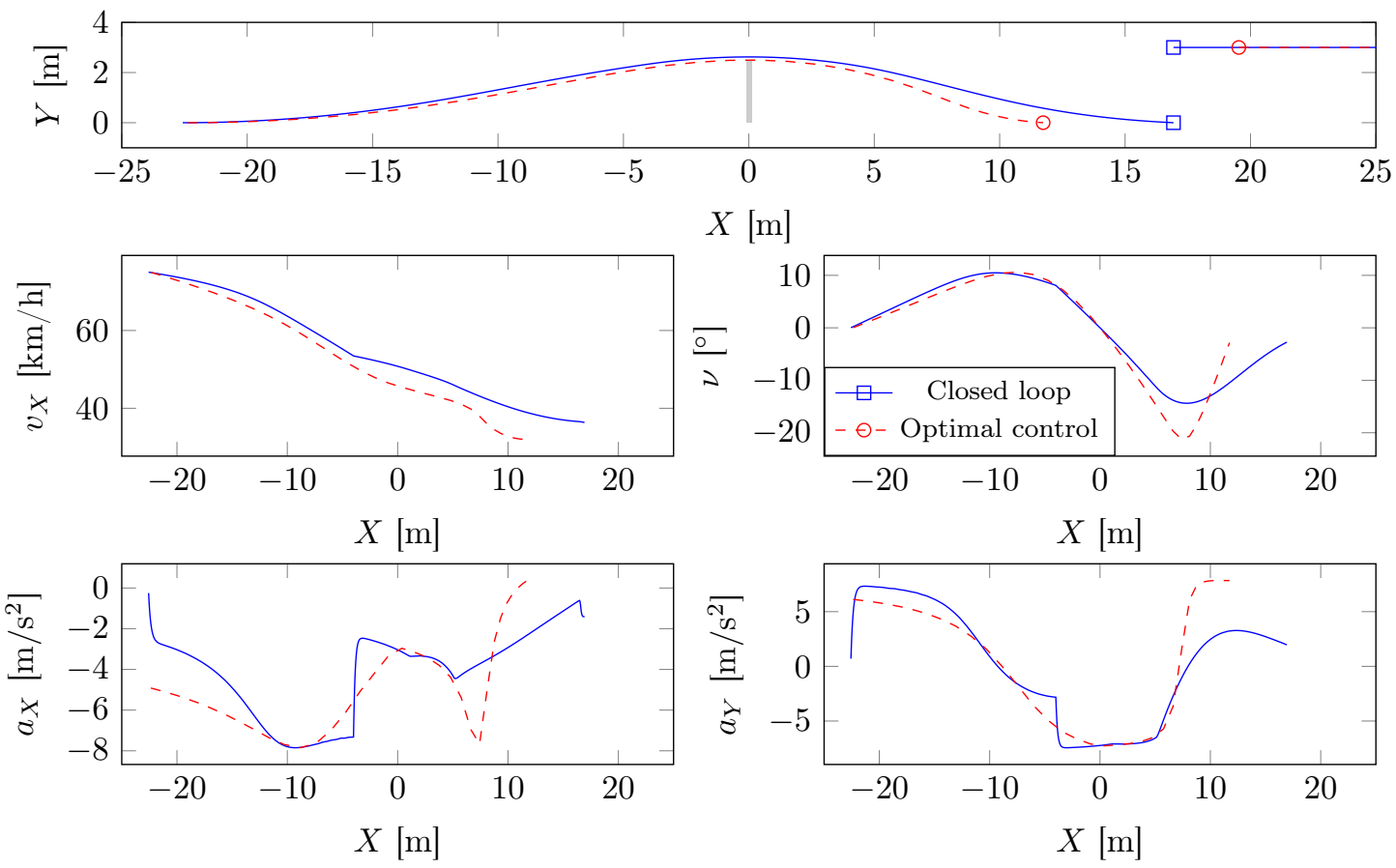

Figure 11 Comparison between closed loop and optimal control for the point mass model in Scenario B. The markers in the path plot represent the location of the vehicles at the end of the manoeuvre.

First, the control allocation scheme that is used to combine the longitudinal acceleration and yaw stability controllers is presented. This control allocation distributes the control inputs to best satisfy the global force and moment demands while taking into account the actuator limitations (amplitude and rate), the vehicle state (understeer/oversteer) and tyre force capacities.

The integrated controller is then implemented and tested in simulation using a gasolineelectric Volvo XC90 vehicle model in CarMaker. Three vehicle setups are considered for comparison: with ESC only (reference), with integrated controller but no propulsion allowed and lastly with the integrated controller and propulsion allowed. The results from the simulation are compared to quantify the distance margin improvement that the integrated controller can achieve in this scenario with and without propulsion.

\subsection{Combining Longitudinal Acceleration and Yaw Stability Control}

While the longitudinal acceleration controller improved the distance margin when used with the point mass model when it was alone, in a real car there will likely be other systems placing - possibly conflicting - demands on the tyre longitudinal forces. One such example is the electronic stability control (ESC) which brakes different wheels to stabilise the vehicle and decelerates the vehicle as a side-effect. It is necessary to ensure that the longitudinal acceleration controller can work with such systems to not only increase the distance margin, but also achieve the goals of these other systems as well. In addition, there may be other restrictions such as actuator limitations, tyre friction limit, etc. which need to be considered when distributing the tyre forces.

In order to ensure the various demands and restrictions are satisfied, control allocation is used to distribute the tyre longitudinal forces. The control allocator takes the global force and moment demands from the various controllers as inputs and distributes the tyre 
forces to satisfy these global demands as best as possible while taking into account the different actuator and tyre force restrictions. The control allocation has been performed using the Quadratic Programming Control Allocation Toolbox (QCAT) ${ }^{2}$ for Simulink using the Weighted Least Squares (WLS) formulation.

In this work, only an ESC is considered for evaluation with the longitudinal acceleration controller. Note that while the actual vehicle (XC90) contains other stability systems such as rollover prevention, they are not implemented in simulation. However, the ESC has been tuned to mimic the combined overall behaviour of all the stability systems in this scenario (but only in this specific scenario) using the test results from Section 2.3. Note that the ESC implemented here is not representative of a industrialized ESC in general. The ESC is modelled using the following equations:

$$
\begin{aligned}
\omega_{\text {err }} & =\omega-\omega_{\text {ref }} \\
M_{z, t g t} & = \begin{cases}-I_{z z} \frac{\omega_{\text {err }}}{\tau_{\omega}} & \left|\omega_{\text {err }}\right| \geq \omega_{\text {thresh }} \\
0 & \text { otherwise }\end{cases}
\end{aligned}
$$

where, $\omega$ and $\omega_{r e f}$ are the actual and reference yaw rates respectively, $I_{z z}$ is the yaw moment of inertia of the vehicle, $\tau_{\omega}$ is the desired yaw response time and $\omega_{\text {thresh }}$ is the yaw rate threshold for the activation of the ESC. The parameters $\tau_{\omega}$ and $\omega_{\text {thresh }}$ are tuned so as to get the ESC to mimic the behaviour of the test vehicle in this specific scenario. The yaw moment target so obtained is then acted upon by the control allocator to apply braking forces on the appropriate wheel.

ESC integration in the control system is needed here since ESCs are typically low level safety-critical controllers that can override other control systems. A common step taken by ESCs while stabilising the vehicle is to cut all propulsion to the wheels which as shown can be detrimental in this scenario. Switching off the ESC is not suitable since this is an on-limit scenario where rapid speed changes can destabilise the vehicle if it is not already unstable. The integrated controller presented here arbitrates between the ESC and the longitudinal acceleration controller as opposed to the ESC simply overriding other controllers.

The structure of this combined controller is shown in Figure 12.

The control allocation problem is then formulated as follows:

$$
\begin{array}{r}
\underset{\boldsymbol{u}}{\arg \min }\left\|\boldsymbol{W}_{\boldsymbol{u}}\left(\boldsymbol{u}-\boldsymbol{u}_{\boldsymbol{d}}\right)\right\|^{2}+\lambda\left\|\boldsymbol{W}_{\boldsymbol{v}}(\boldsymbol{B} \boldsymbol{u}-\boldsymbol{v})\right\|^{2} \\
\text { subj. to } \quad-\boldsymbol{u} \leq \boldsymbol{u} \leq{ }^{+} \boldsymbol{u} \\
-\dot{\boldsymbol{u}} \leq \dot{\boldsymbol{u}} \leq{ }^{+}{ }_{\boldsymbol{u}}
\end{array}
$$

\footnotetext{
${ }^{2}$ The toolbox is available for free download at https://se.mathworks.com/matlabcentral/fileexchange/4609-qcat
} 


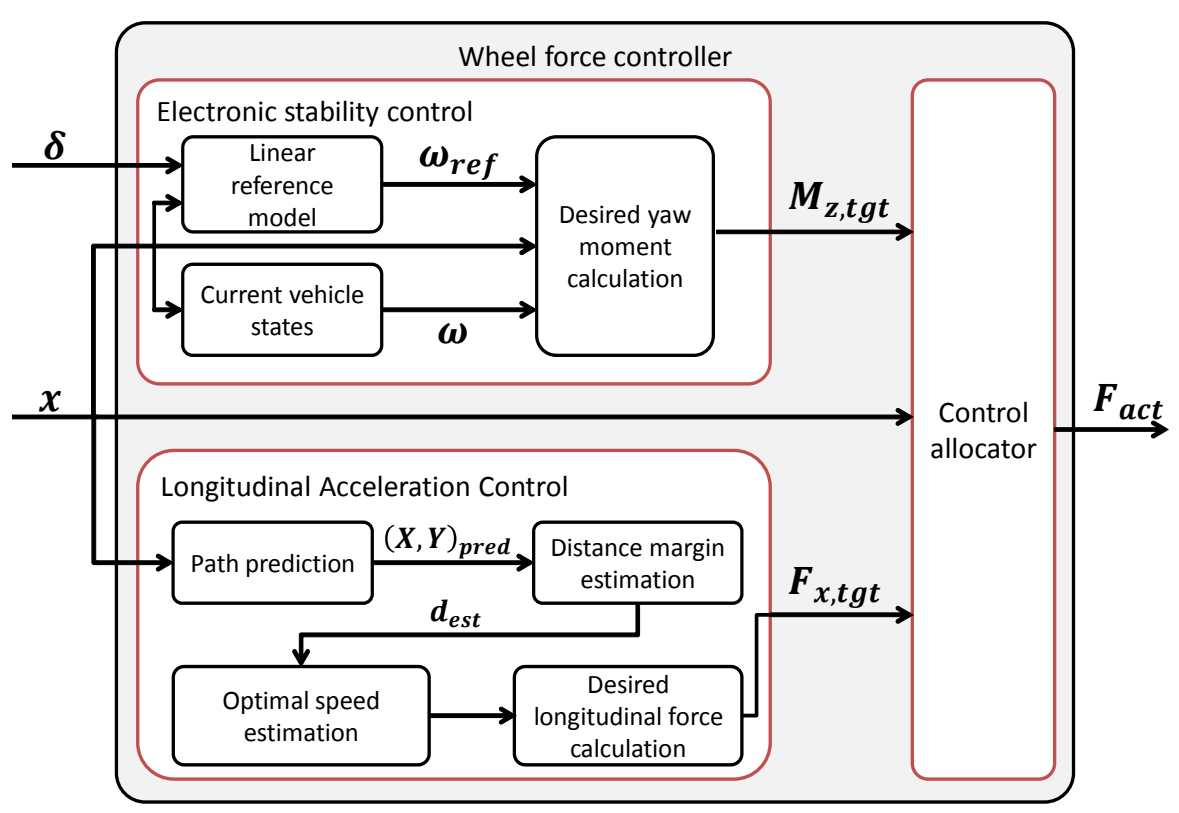

Figure 12 Structure of the integrated controller.

where,

$$
\begin{aligned}
& \boldsymbol{W}_{\boldsymbol{u}}=\boldsymbol{K}_{\dot{u}} \boldsymbol{K}_{\boldsymbol{F}_{z}}
\end{aligned}
$$

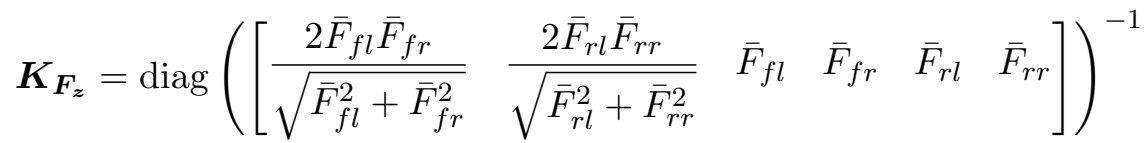

$$
\begin{aligned}
& \boldsymbol{K}_{\dot{\boldsymbol{u}}}=\operatorname{diag}\left(\left[\begin{array}{llllll}
K_{\text {eng }} & K_{m t r} & K_{b r k, f l} & K_{b r k, f r} & K_{b r k, r l} & K_{b r k, r r}
\end{array}\right]\right)^{-1} \\
& \bar{F}_{i j}=f_{i} \mu \hat{F}_{z, i j} \\
& \hat{F}_{z, i j}=m g \frac{L-l_{i}}{2 L}+(-1)^{i} \sigma_{x} m a_{x}+(-1)^{j} \sigma_{y} m a_{y} \\
& K_{i}=\left(\frac{{ }^{+} \dot{u}_{i}^{2}+{ }^{-} \dot{u}_{i}^{2}}{\sum_{j=1}^{n}+\dot{u}_{j}^{2}+{ }^{-} \dot{u}_{j}^{2}}\right)^{1 / 2} i \in\{e n g, m t r, b r k, f l, b r k, f r, b r k, r l, b r k, r r\} \\
& \boldsymbol{u}_{\boldsymbol{d}}=\mathbf{0}_{6 \times 1} \\
& \boldsymbol{W}_{\boldsymbol{v}}=\operatorname{diag}\left(\left[\begin{array}{ll}
1 & 5
\end{array}\right]\right) \\
& \boldsymbol{B}=\left[\begin{array}{cccccc}
1 & 1 & 1 & 1 & 1 & 1 \\
0 & 0 & -w / 2 & w / 2 & -w / 2 & w / 2
\end{array}\right] \\
& \boldsymbol{u}=\left[\begin{array}{llllll}
F_{\text {eng }} & F_{m t r} & F_{b r k, f l} & F_{b r k, f r} & F_{b r k, r l} & F_{b r k, r r}
\end{array}\right]^{T} \\
& \boldsymbol{v}=\left[\begin{array}{ll}
F_{x, t g t} & M_{z, t g t}
\end{array}\right]^{T}
\end{aligned}
$$

$\boldsymbol{K}_{\boldsymbol{F}_{z}}$ is the weight matrix that is used in order to minimise the actuator and the tyre workload. Minimum actuator workload ensures reduced energy consumption from the actuators while minimum tyre workload ensures reduced tyre wear, improved grip and energy dissipation in the tyres. The combined actuator-tire workload can be expressed as 
the squared sum of the normalised actuator force at each tyre as follows:

$$
\begin{aligned}
J_{w r k l d} & =\left(\frac{F_{\text {eng }} / 2}{\bar{F}_{f l}}\right)^{2}+\left(\frac{F_{\text {eng }} / 2}{\bar{F}_{f r}}\right)^{2}+\left(\frac{F_{b r k, f l}}{\bar{F}_{f l}}\right)^{2}+\left(\frac{F_{b r k, f r}}{\bar{F}_{f r}}\right)^{2} \\
& +\left(\frac{F_{m t r} / 2}{\bar{F}_{r l}}\right)^{2}+\left(\frac{F_{m t r} / 2}{\bar{F}_{r r}}\right)^{2}+\left(\frac{F_{b r k, r l}}{\bar{F}_{r l}}\right)^{2}+\left(\frac{F_{b r k, r r}}{\bar{F}_{r r}}\right)^{2}
\end{aligned}
$$

Since the first term of the control allocation objective function (Equation (26)) serves to perform a weighted squares minimisation of the actuator forces, it can be used to minimise the actuator-tyre workload workload as well. Hence, collecting the terms together and taking the coefficients of the actuator forces in Equation (38), gives the terms of the $\boldsymbol{K}_{\boldsymbol{F}_{z}}$ matrix which becomes part of $\boldsymbol{W}_{\boldsymbol{u}}$. Ideally, the tyre workload should also take into account the lateral forces at the tyres, but since $F_{y}$ is unknown for the tyres (and are not easy to estimate, especially when operating in the non-linear area of the tyres), we use only the longitudinal tyre force here.

$\bar{F}_{i j}$ here is the tyre force capacity of wheel $i j$. This is computed by taking the product of the estimated wheel load, the friction and a factor $f_{i}$ which is used to account for understeer/oversteer. For the front axle, this factor goes from one to zero as the vehicle begins to understeer. Similarly, for the rear axle, it goes from one to zero as the vehicle begins to oversteer.

While the $K_{F_{z}}$ matrix serves to minimise the tyre-actuator workload, it does not take into account the capabilities of the different actuators. For instance, if excess control is allocated to a slow actuator, the performance of the controller can deteriorate. To account for this, the matrix $\boldsymbol{K}_{\dot{\boldsymbol{u}}}$ is introduced. The goal of this matrix is to prioritize using faster actuators over slower ones.

The priority for the actuators is established using the $K_{i}$ term. $K_{i}$ is computed simply as the rate limit of an actuator normalised by the $\ell^{2}$-norm of rate limits for all actuators acting on that wheel. However, since some actuators (engine) have different rising and falling rate limtis, the root mean square (RMS) of the rising and falling rates are used as the rate limits for the actuator. Here, the subscripts eng, $m t r, b r k, f l, b r k, f r, b r k, r l$ and $b r k, r r$ represent the actuators engine, motor, brakes on the front left, front right, rear left and rear right respectively.

The $\boldsymbol{K}_{\boldsymbol{F}_{\boldsymbol{z}}}$ and $\boldsymbol{K}_{\dot{\boldsymbol{u}}}$ matrices are then multiplied to yield the $\boldsymbol{W}_{\boldsymbol{u}}$ matrix that serves to minimise the tyre-actuator workload while also prioritising faster actuators to improve performance.

The $\boldsymbol{W}_{\boldsymbol{v}}$ matrix determines the trade-off between meeting the global longitudinal force target $\left(F_{x, t g t}\right)$ versus the global yaw moment target $\left(M_{z, t g t}\right)$. The matrix was determined by trial and error starting from an identity matrix and gradually increasing the yaw moment component until the vehicle managed to complete the manoeuvre in a stable manner with good distance margin.

\subsection{Simulations show Robust Increases in Distance Margin with Integrated Control}

Detail simulations were then run in CarMaker using a validated Volvo XC90 vehicle model [18] provided by Volvo Car Corporation. The driver is represented using a steering controller from [17] which is shown to work well in limit conditions. The preview parameters are tuned to yield a steering profile with characteristics similar to that seen in the experiments.

Figures 13 and 14 show the simulation results for scenario A and B respectively. In 
each scenario, three cases are considered: first with ESC only, next with the integrated controller but using only the brakes, and finally with the integrated controller using the brakes and the propulsion actuators as well (motor and engine).
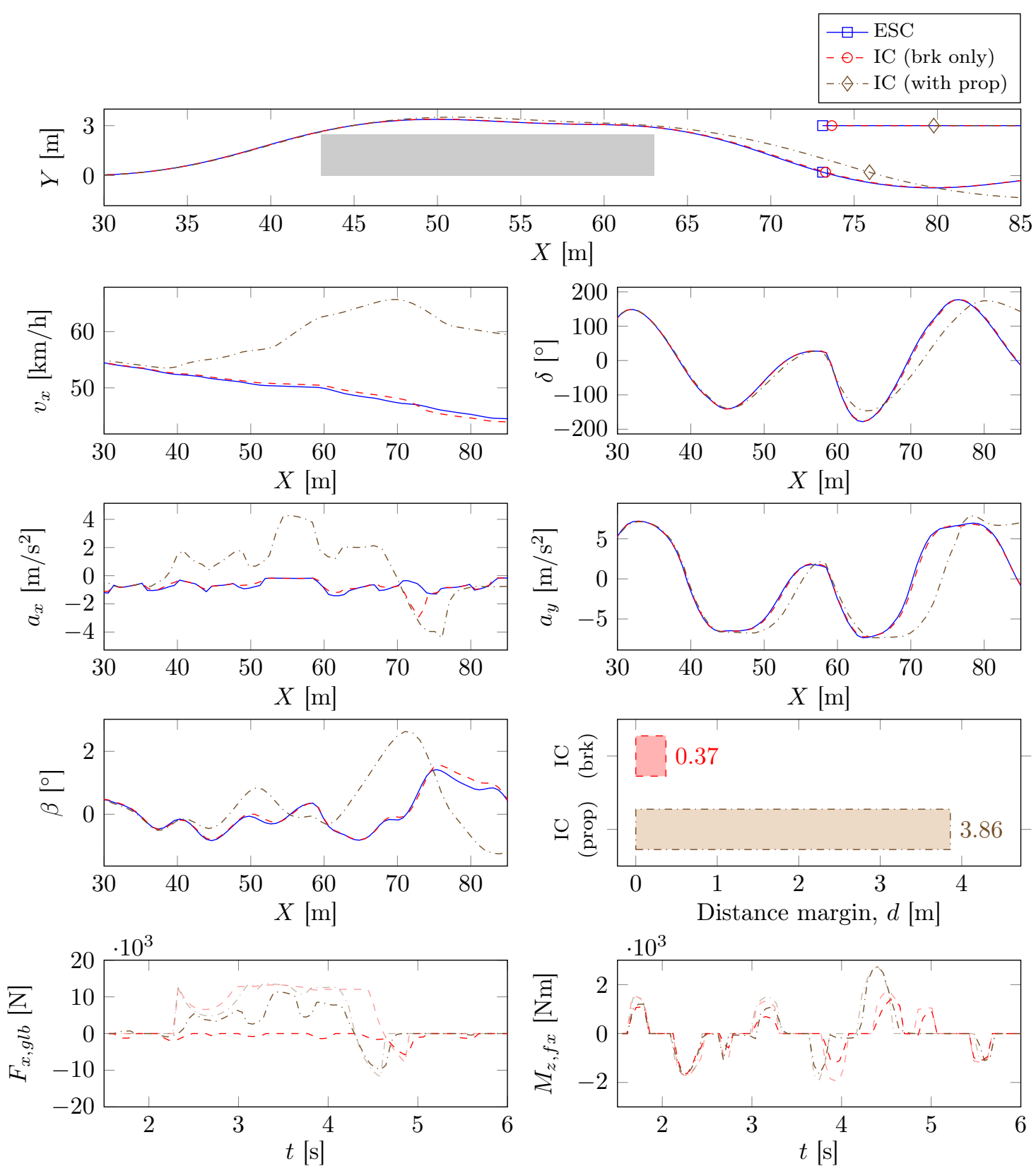

Figure 13 Performance of the integrated controller in Scenario A. ESC = Electronic Stability Control, IC = Integrated Control. The markers in the path plot represent the location of the vehicles at the end of the manoeuvre. In the distance margin plot, the improvements in distance margin achieved by the integrated controllers over the case with ESC only are shown. The last row shows the global force and moments demanded (greyed out plots) and applied by the controllers. For clarity, the 'ESC' case is omitted in these plots.

In Figure 13, it can be seen that using the integrated controller with brakes only marginally increases the distance margin. Since acceleration is desirable in this scenario, the lack of propulsion severely limits the distance margin increase that can be achieved. The small distance margin increase is achieved by limiting the differential braking where 

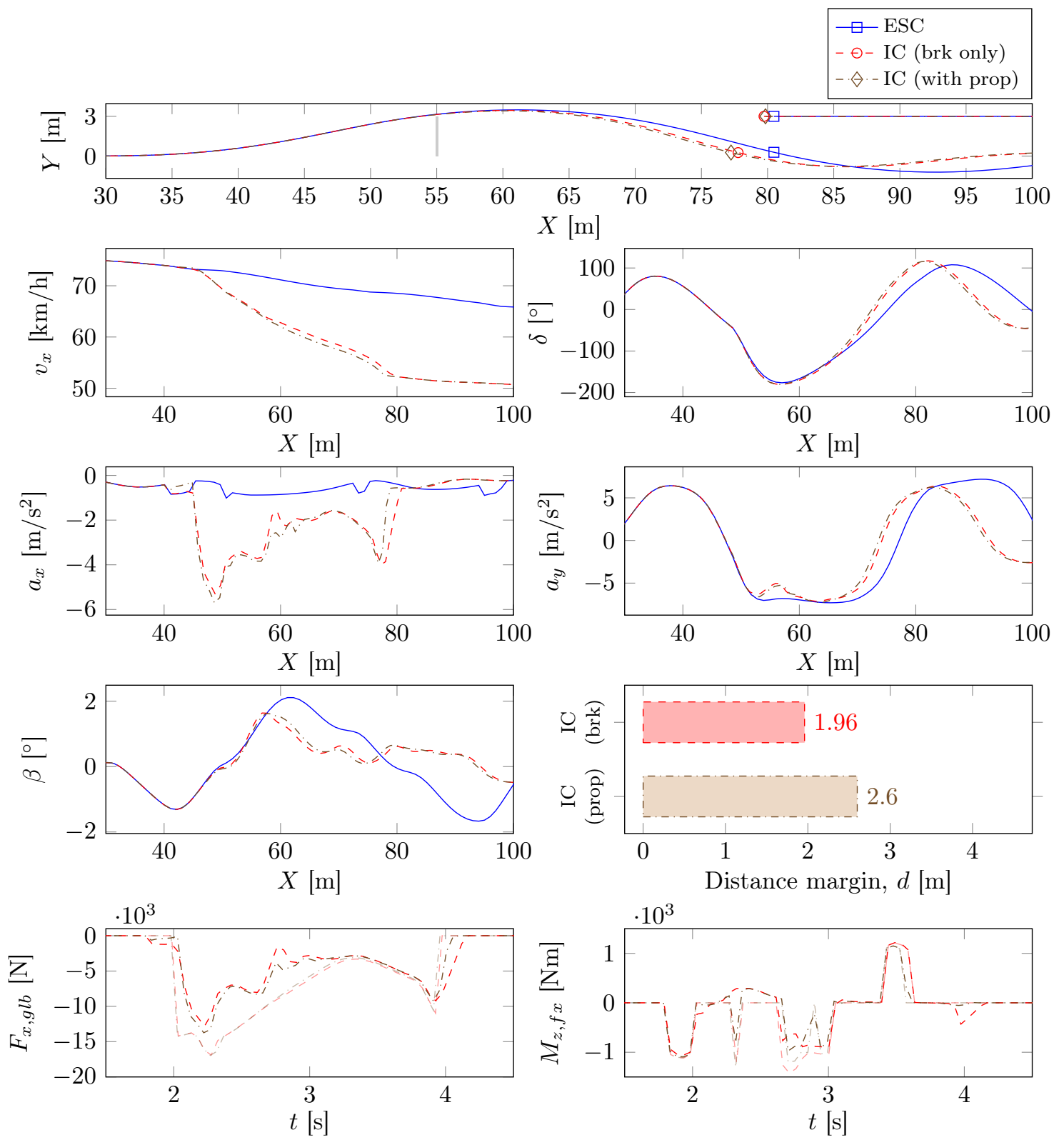

Figure 14 Performance of the integrated controller in Scenario B. ESC = Electronic Stability Control, IC = Integrated Control. The markers in the path plot represent the location of the vehicles at the end of the manoeuvre. In the distance margin plot, the improvements in distance margin achieved by the integrated controllers over the case with ESC only are shown. The last row shows the global force and moments demanded (greyed out plots) and applied by the controllers. For clarity, the 'ESC' case is omitted in these plots.

possible in order to reduce the deceleration side-effect from the same. This can be observed in the velocity and the longitudinal acceleration plots where the amplitudes with integrated control are marginally smaller until the obstacle is passed. The impact of the reduced differential braking on the yaw stability of the vehicle is also marginal and cannot be observed in the plots. Additionally, little difference can be seen in the steering wheel angle, lateral acceleration and yaw rates of these two cases. Additionally, the demanded and the delivered global force and moments can also be seen in the last row plots of Figure 13. Here, the trade-off the control allocation makes between delivering the yaw moment and the not slowing the vehicle down can be seen. 
When propulsion is allowed however, a significant increase in distance margin can be seen despite the vehicle taking a longer distance to complete the manoeuvre. In this case, the vehicle accelerates in order to complete the manoeuvre in a shorter time due to the faster oncoming vehicle. Note that the longitudinal force demand is not limited in this implementation and in a more real-world implementation, the acceleration allowed can be restricted, which however would also reduce the distance margin improvement that can be achieved. The increased distance margin in this case comes at the cost of reduced yaw stability and path following performance as can be seen from the side-slip and path plots respectively. This trade-off between yaw stability and distance margin can be controlled by appropriately choosing the $\boldsymbol{W}_{\boldsymbol{v}}$ matrix. The yaw rate and the steering amplitudes are reduced slightly towards the end of the manoeuvre which can be explained by the increased speed. In the global force and moment plots of Figure 13, it can be seen that when propulsion is allowed, the trade-off between longitudinal force and yaw moment is also improved resulting in the delivered yaw moments being much closer to the demanded. The resulting control allocated to the different actuators acting on each tyre can be seen in Figure 15.
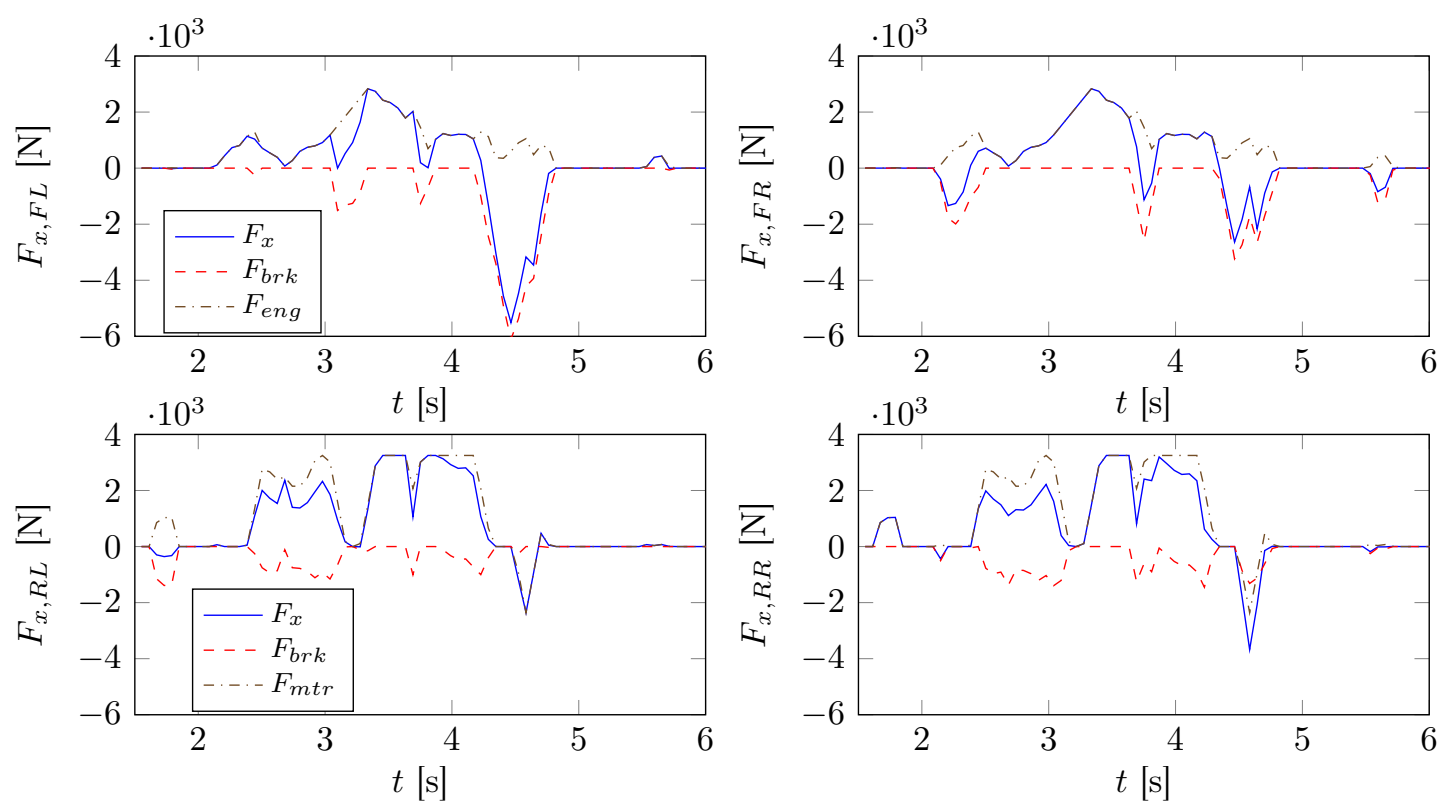

Figure 15 Actuator forces on the tyres in Scenario A

In Figure 14 (scenario B), it can be seen that even when only the brake actuators are available, the integrated control results in a big increase in the distance margin compared to the conventional ESC control. This is due to deceleration being desirable in this case for which the brake actuators are adequate. With the integrated control, a much bigger drop in speed and deceleration is seen. This in turn results in slightly higher yaw rates and steering wheel angles being required in the end due to the reduced speed. However, the reduced speed results in much improved stability (side-slip plot) and path following where the vehicle manages to return to the original lane in a shorter distance compared to the case with ESC. Note however, that the vehicle takes a longer time to complete the manoeuvre with the integrated control (see oncoming vehicle path), which however is of little consequence since the oncoming vehicle travels relatively slowly in this case.

When propulsion is allowed in this scenario, a small (but noticeable) increase in distance margin is seen. This can be attributed partly to the improved trade-off between yaw 

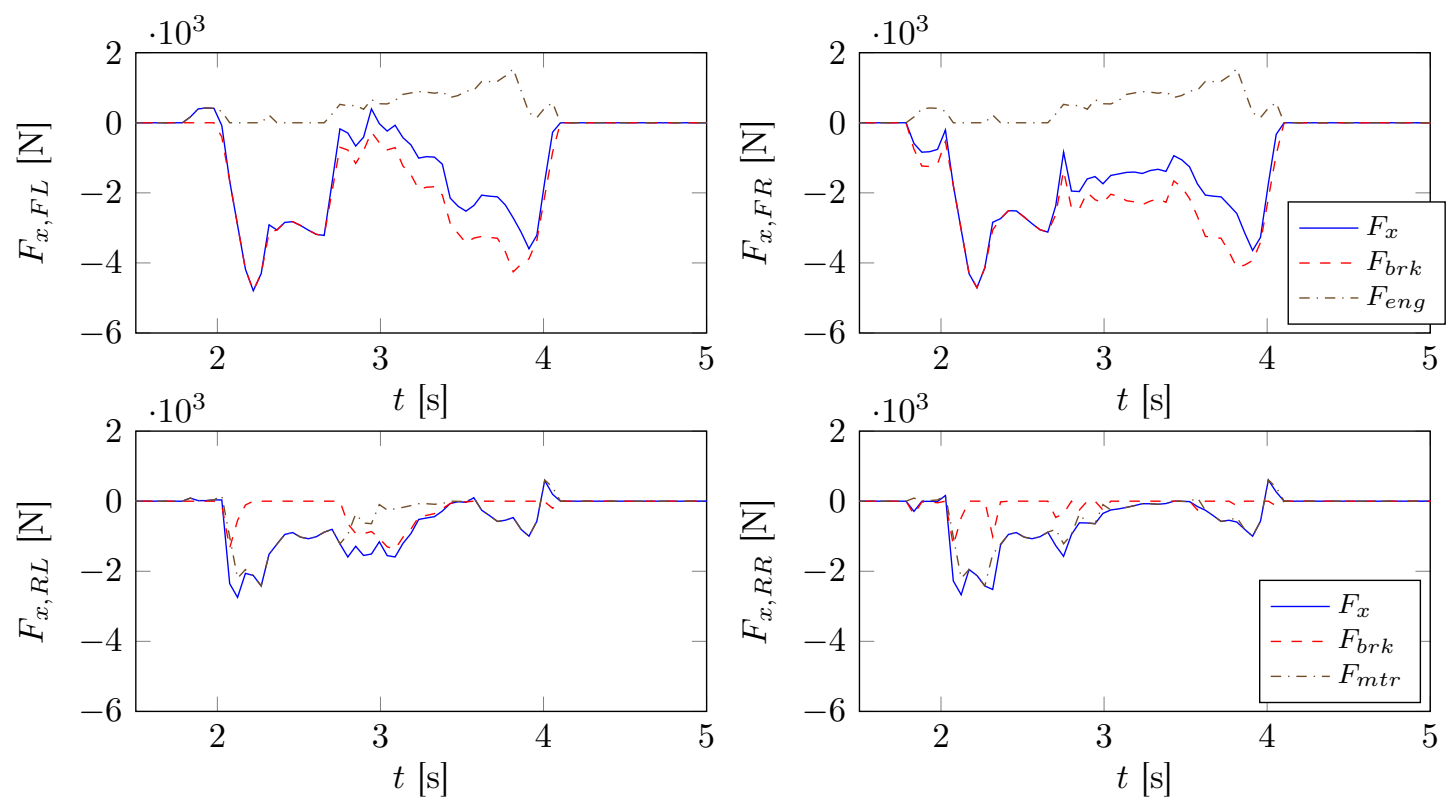

Figure 16 Actuator forces on the tyres in Scenario B

moment and longitudinal force that can be achieved by the control allocation (see last row plots of Figure 14). From the control allocation plots in Figure 16, it can be seen that propulsion is used on the front axle even though acceleration is not desirable in this case. This is done in order to generate larger yaw moment magnitudes across the axle. This improved trade-off results in slightly improved path following and slightly higher decelerations which in turn results in the vehicle completing the manoeuvre in a shorter distance. The difference in the steering wheel angles, yaw rates, lateral acceleration and side slips are marginal compared to the case with brakes only.

The distance margin plots of Figures 13 and 14 show the distance margin improvements achieved by the controllers in the two scenarios. As expected, integrated control with brakes alone can make a big difference in scenario B whereas it makes little difference in scenario A. In contrast, propulsion makes a big difference in scenario A and little in scenario B over the case with brakes only. In either case, addition of propulsion increases the benefit achievable albeit to different extents.

Lastly, preliminary simulations were run using the controller with different steering preview parameters to confirm that the controller performs robustly for different steering inputs. A more detailed analysis of the controller's performance with regards to driver interaction, steering characteristics, etc. will need be performed in the future.

\section{Conclusions}

The task of vehicle dynamic control for mitigation of collision risk with oncoming vehicles during evasive manoeuvres is considered in the present work.

An optimal control analysis of this manoeuvre highlighted the importance of speed in this particular accident scenario. The global X-distance between the host and the oncoming vehicle at the end of the manoeuvre is used as a measure of the collision risk with the oncoming vehicle and is called the distance margin. The relationship between various scenario parameters and speed through the manoeuvre to minimise collision risk was studied. A characteristic parameter was discovered which was seen to have a strong 
correlation to the reduction in collision risk that can be achieved by controlling the longitudinal force during the manoeuvre. The influence of speed on the distance margin in this manoeuvre was determined to be due to a trade-off between distance travelled and time duration of the manoeuvre. The duration becomes particularly important if the oncoming vehicle is travelling at a high speed relative to the host vehicle or in case of a long obstacle.

This result from the optimal control analysis was preliminarily validated in experiments conducted using a Volvo XC90 test vehicle wherein the speed was controlled manually, i.e., open-loop, by the driver. The resulting distance margins followed the trend as predicted by the optimal control results, i.e., speed increases were seen to benefit the cases where the characteristic parameter was small.

Based on the insights gained from the optimal control analysis, a closed-loop longitudinal acceleration controller is proposed. As a base check of the control strategy, this controller is first validated using a point mass model and compared to the optimal control results. This was then followed by detailed simulations in CarMaker using a validated XC90 vehicle model with an integrated controller consisting of a yaw stability controller representing a conventional ESC, and the longitudinal acceleration controller. It was seen that significant increases in distance margins could be achieved in scenario B (short obstacle) using brakes alone whereas in scenario A (long obstacle), propulsion was needed to make a big difference. It is interesting to note that, in all cases, addition of integrated control with brakes increased the distance margin over conventional ESC, and addition of propulsion increased the distance margin even further over the cases of brakes only.

In future work, further verification of the robustness of the integrated controller with respect to different types of steering inputs needs to be done. Initial results in CarMaker simulations have already shown that higher benefits in terms of distance margins are achievable when the steering effort is very high (overactive driver) or very low (inactive driver). More generally, the driver interaction aspect of the controller needs to be investigated. Full-vehicle tests with the closed-loop controller implementation are also planned for further validation of the controller performance and demonstration of the real-time capability of the proposed controller.

\section{References}

[1] Singh S. Driver attributes and rear-end crash involvement propensity. NHTSA; 2003. Technical report DOT HS 809540.

[2] Fildes B, Keall M, Bos N, Lie A, Page Y, Pastor C, Pennisi L, Rizzi M, Thomas P, Tingvall C. Effectiveness of low speed autonomous emergency braking in real-world rear-end crashes. Accident Analysis \& Prevention. 2015 Aug;81:24-29; Available from: http://www.sciencedirect.com/science/ article/pii/S0001457515001116.

[3] Schittenhelm H. Advanced Brake AssistReal World effectiveness of current implementations and next generation enlargements by Mercedes-Benz. In: Proceedings of the 23rd International Technical Conference on the Enhanced Safety of Vehicles (ESV); May. Seoul, South Korea; 2013. Available from: http://www-nrd.nhtsa.dot.gov/pdf/ESV/esv23/23ESV-000194.pdf.

[4] Kusano K, Gabler H. Safety Benefits of Forward Collision Warning, Brake Assist, and Autonomous Braking Systems in Rear-End Collisions. IEEE Transactions on Intelligent Transportation Systems. 2012 Dec;13(4):1546-1555.

[5] Brännström M, Coelingh E, Sjöberg J. Model-Based Threat Assessment for Avoiding Arbitrary Vehicle Collisions. IEEE Transactions on Intelligent Transportation Systems. 2010;11(3):658-669.

[6] Shah J. Development and Control of Evasive Steer Assist Using Rear Wheel Steering. Warrendale, PA: SAE International; 2015. Report No.: 2015-26-0004; Available from: http://www.sae.org/ technical/papers/2015-26-0004.

[7] Dang DT, Desens DJ, Franke DU, Gavrila PDD, Schäfers L, Ziegler DW. Steering and Evasion Assist. 
In: Eskandarian A, editor. Handbook of Intelligent Vehicles. Springer London; 2012. p. 759-782; dOI: 10.1007/978-0-85729-085-4_29; Available from: http://link.springer.com/referenceworkentry/ 10.1007/978-0-85729-085-4_29.

[8] Keller CG, Dang T, Fritz H, Joos A, Rabe C, Gavrila DM. Active pedestrian safety by automatic braking and evasive steering. Intelligent Transportation Systems, IEEE Transactions on. 2011; 12(4):1292-1304.

[9] Yang D, Jonasson M, Halleröd T, Johansson R. Evaluation of an Evasive Manoeuvre Assistance System at Imminent Side Collisions. In: Proceedings of the 13th International Symposium on Advanced Vehicle Control; Sep. Munich, Germany; 2016. Available from: http://www.avec16.com/ images/papers/37480.pdf.

[10] Kelly Pleskot. New Volvo XC60 Will Steer You Away From Potential Accidents. 2017 Feb; Available from: http://www.motortrend.com/news/new-volvo-xc60-will-steer-away-potential-accidents/.

[11] Mitrache V. 2017 Mercedes-Benz E-Class Evasive Steering Assist Function Explained. 2016 Jul; Available from: https://www.autoevolution.com/news/ 2017-mercedes-benz-e-class-evasive-steering-assist-function-explained-109292.html.

[12] Isermann R, Mannale R, Schmitt K. Collision-avoidance systems PRORETA: Situation analysis and intervention control. Control Engineering Practice. 2012 Nov;20(11):1236-1246; Available from: http://linkinghub.elsevier.com/retrieve/pii/S0967066112001256.

[13] Perez J, Milanes V, Onieva E, Godoy J, Alonso J. Longitudinal fuzzy control for autonomous overtaking. In: Mechatronics (ICM), 2011 IEEE International Conference on. IEEE; 2011. p. 188193; Available from: http://ieeexplore.ieee.org/xpls/abs_all.jsp?arnumber $=5971279$.

[14] Usman G, Kunwar F. Autonomous vehicle overtaking-an online solution. In: Automation and Logistics, 2009. ICAL'09. IEEE International Conference on. IEEE; 2009. p. 596-601; Available from: http://ieeexplore.ieee.org/xpls/abs_all.jsp?arnumber $=5262854$.

[15] Ngai DC, Yung NH. Automated vehicle overtaking based on a multiple-goal reinforcement learning framework. In: Intelligent Transportation Systems Conference, 2007. ITSC 2007. IEEE. IEEE; 2007. p. 818-823; Available from: http://ieeexplore.ieee.org/xpls/abs_all.jsp?arnumber $=4357682$.

[16] Shamir T. How should an autonomous vehicle overtake a slower moving vehicle: design and analysis of an optimal trajectory. IEEE Transactions on Automatic Control. 2004 Apr;49(4):607-610.

[17] Klomp M, Olsson K, Sandberg C. Nonlinear steering control for limit handling conditions using preview path curvature. International Journal of Vehicle Autonomous Systems. 2014 Jan;12(3):266283; Available from: http://dx.doi.org/10.1504/IJVAS.2014.063043.

[18] Angelis S, Johnsson A, Klomp M, Hansson R, Katzourakis D. Virtual brake software release. In: The Dynamics of Vehicles on Roads and Tracks. CRC Press; 2016. p. 521-532; dOI: 10.1201/b21185-57 DOI: 10.1201/b21185-57; Available from: http://www.crcnetbase.com/doi/abs/10.1201/b21185-57.

\section{Appendix A. Nomenclature}

Symbol
$a_{x}, a_{y}$
$a_{X}, a_{Y}$
$b r k, f l$
$b r k, f r$
$b r k, r l$
$b r k, r r$
$d$
$d_{0}$
$d_{t r}$
$d_{t r, h}$

\section{Description}

Longitudinal and lateral acceleration in vehicle frame

Global X and Y-acceleration

Subscript representing the front left brake actuator

Subscript representing the front right brake actuator

Subscript representing the rear left brake actuator

Subscript representing the rear right brake actuator

Global X-distance margin - distance between the host and the

bullet (oncoming) vehicle at the end of the manoeuvre

Global X-distance between the host and the bullet vehicle at the beginning of the manoeuvre

Global X-distance travelled by the host and the bullet vehicle over the duration of the manoeuvre

Global X-distance travelled by the host vehicle over the duration of the manoeuvre 


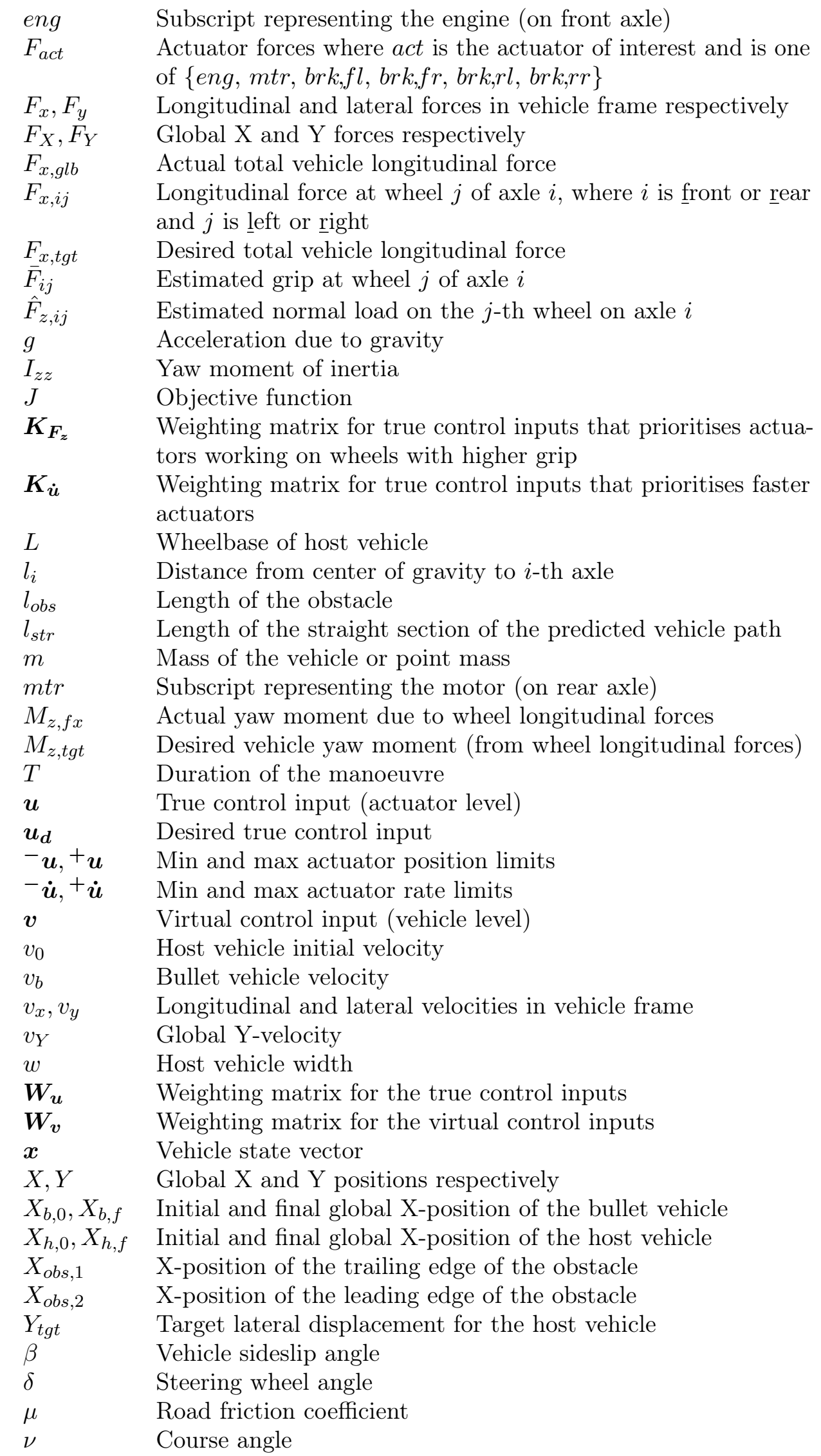
of $\{e n g, m t r, b r k, f l, b r k, f r, b r k, r l, b r k, r r\}$

$F_{x}, F_{y}$

$F_{x, g l b}$

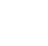


$\sigma_{x}, \sigma_{y} \quad$ Longitudinal and lateral load transfer coefficients respectively $\tau_{i} \quad$ Estimated time durations to complete $i$-th section of the path $\tau_{\omega} \quad$ Desired yaw response time for the ESC

$\omega \quad$ Yaw rate

$\omega_{\text {ref }} \quad$ Reference yaw rate (from reference model)

$\omega_{\text {thresh }} \quad$ Yaw rate error threshold for ESC activation

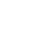

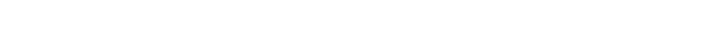

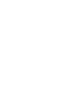

